\title{
Influence of low and decreasing food levels on Daphnia-algal interactions: Numerical experiments with a new dynamic energy budget model
}

\author{
F. Peeters ${ }^{a}, *$, J. Li ${ }^{a}$, D. Straile ${ }^{a}$, K.-O. Rothhaupt ${ }^{a}$, J. Vijverberg ${ }^{b}$ \\ a Limnological Institute, University of Konstanz, Mainaustrasse 252, D-78464 Konstanz, Germany \\ ${ }^{b}$ Netherlands Institute of Ecology (NIOO-KNAW), Centre for Limnology, Rijksstraatweg 6, 3631 AC Nieuwersluis, The Netherlands
}

Keywords:

Predator-prey interaction

Population dynamics

Starvation

DEB-model

Daphnia

\begin{abstract}
A B S T R A C T
Based on numerical experiments with a new physiologically structured population model we demonstrate that predator physiology under low food and under starving conditions can have substantial implications for population dynamics in predator-prey interactions. We focused on Daphnia-algae interactions as model system and developed a new dynamic energy budget (DEB) model for individual daphnids. This model integrates the $\kappa$-rule approach common to net assimilation models into a netproduction model, but uses a fixed allocation of net-productive energy in juveniles. The new DEB-model agrees well with the results of life history experiments with Daphnia. Compared to a pure $\kappa$-rule model the new allocation scheme leads to significant earlier maturation at low food levels and thus is in better agreement with the data. Incorporation of the new DEB-model into a physiologically structured population model using a box-car elevator technique revealed that the dynamics of Daphnia-algae interactions are highly sensitive to the assumptions on the energy allocation of juveniles under low food conditions. Additionally we show that also other energy allocation rules of our DEB-model concerning decreasing food levels and starving conditions at the individual level have strong implications for Daphnia-algae interactions at the population level. With increasing carrying capacity of algae a stable equilibrium with coexistence of Daphnia occurs and algae shifts to limit cycles. The amplitudes of the limit cycles increase with increasing percentage of sustainable weight loss. If a $K$-rule energy allocation is applied to juveniles, the stable equilibrium occurs for a much narrower range of algal carrying capacities, the algal concentration at equilibrium is about 2 times larger, and the range of algae carrying capacities at which daphnids become extinct extends to higher carrying capacities than in the new DEB-model. Because predator-prey dynamics are very sensitive to predator physiology under low food and starving conditions, empirical constraints of predator physiology under these conditions are essential when comparing model results with observations in laboratory experiments or in the field.
\end{abstract}

\section{Introduction}

Periods of food scarcity associated with starvation have been shown to strongly influence the population dynamics of many populations in freshwater, marine and terrestrial ecosystems (Tessier, 1986; Tyler et al., 2008; van de Wolfshaar et al., 2008; Robb et al., 2008). In biomass based Lotka-Volterra type predator-prey interactions the predators typically experience decreasing food levels during a substantial amount of time in each predator-prey cycle. The cyclic behaviour modelled and observed in many predator-prey interactions therefore depends on the response of the predator to decreasing food levels and starving condi-

\footnotetext{
* Corresponding author. Tel.: +49 753188 3459; fax: +497531 883533.

E-mail address: frank.peeters@uni-konstanz.de (F.Peeters).
}

tions. Hence, a fundamental understanding and simulation of predator-prey interactions requires a realistic description of predator physiology under ample food supply and food shortage but also under decreasing food levels. Decreasing food levels can result in a principal change in energy allocation in individuals (e.g. Bradley et al., 1991b; Kirk, 1997) and cause starvation, defined here as the condition when energy assimilation is not sufficient to satisfy maintenance. The occurrence of starvation does not only depend on the food level but also on the size of the individual because assimilation and maintenance are size dependent. At constant or rising food levels individuals do not grow to a size at which they cannot satisfy maintenance (provided that other external factors affecting somatic growth, e.g. temperature, remain constant). However, when food levels decrease, individuals that have grown to large size at the previously high food level may suffer from starvation, because the energy assimilated at lower food levels may not satisfy their maintenance. Survival and reproduction of individuals 
thus not only depends on the food level itself but also on the food level history. Hence, the growth rate of a predator population in a predator-prey system does not simply depend on food concentration as is typical for biomass models but it is asymmetric with respect to the direction of change in the food level.

The key herbivores of many freshwater ecosystems, water flees of the genus Daphnia, are known to exhibit cyclic dynamics during the vegetation period (McCauley and Murdoch, 1990) in which they strongly suppress their food sources (Lampert et al., 1986). This implies that Daphnia typically experiences periods of declining and low food levels in situ. Consequently, the impact of decreasing food levels on Daphnia physiology and its implication for Daphnia population dynamics is a key factor for the understanding of Daphnia-algae interactions in lakes. Most laboratory experiments investigating the physiology of organisms are performed under constant food conditions. Although these experiments are highly valuable and support physiological insights required in the simulation of population growth under constant or increasing food levels, the information may not be sufficient to allow physiologically based simulations of predator-prey interactions. In studies investigating the implication of food shortage on Daphnia often only the extreme condition of well nourished organisms being exposed to zero food is considered (e.g. Bradley et al., 1991a; Tessier et al., 1983).

Here we investigate the implications of low food and starving physiology on predator-prey dynamics focussing on Daphnia-algae interactions as model system. We present a model that considers low food and decreasing food levels within the framework of a dynamic energy budget model (DEB-model). Thereby, we demonstrate how different assumptions on Daphnia physiology under low food and reducing food levels affect predator-prey interactions in physiologically structured population models. The model combines an individual level model based on a DEB approach (Kooijman, 1986, 2001; Lika and Nisbet, 2000; McCauley et al., 1990 ; Noonburg et al., 1998) with a cohort based population model that is solved using the box-car elevator technique (DeRoos et al., 1992) that was modified to consider length and weight as independent state variables. The individual model incorporates the $\kappa$-rule assumption employed in net assimilation models (Kooijman, 2000, 2001; Muller and Nisbet, 2000; Rinke and Vijverberg, 2005) into a net-production model (Nisbet et al., 2004; Noonburg et al., 1998; Vanoverbeke, 2008) by using a new approach to dynamically allocate energy between somatic growth and reproduction that is based mainly on energy fluxes rather than on the physiological state of the organism. Energy allocation in juveniles is designed specifically such that maturating daphnids have invested the same maturation cost independent of the history of food supply. The concepts developed and their consequences for the physiological state of the individual and for the population dynamics are exemplified using mainly Daphnia galeata but also Daphnia pulex as model organisms. Furthermore, we show that our approach is able to reproduce Daphnia growth and reproductive trajectories also at the lowest food concentrations used in the experiments by Rinke and Vijverberg (2005), Urabe (1988) and Nisbet et al. (2004).

In the following we first provide a brief summary on different types of DEB-models and their limitations and then present the modifications we made to the energy allocation rules. Thereby we focus on two different levels of reduced food: (1) Food levels at which maintenance can be satisfied but at which the remaining energy is less than that required for reproduction by the $\kappa$-rule assumption of e.g. Kooijman (2000, 2001); (2) Food levels at which the organisms cannot satisfy their maintenance and thus loose weight due to starvation. After the presentation of the new DEBmodel we compare model simulations with life history data. Finally we demonstrate that the energy allocation at the individual level has significant consequences for Daphnia-algae interactions by providing the results from model simulations of the Daphnia popu- lation dynamics using a structured population model than includes the new DEB-model.

The model equations of the individual and of the structured population model are summarized in Table 1 and in Appendix Table A3, respectively. The parameters used in the simulations of $D$. galeata and $D$. pulex are listed in Tables $A 1$ and $A 2$ of Appendix, respectively.

\section{Development of the individual level model}

\subsection{General concepts of dynamic energy budget models (DEB)}

Dynamic energy budget models have been employed to describe the physiology of organisms in age or size structured population models. Specific attention has been given to models of Daphnia (e.g. McCauley et al., 1990b; Noonburg et al., 1998; Kooijman, 1986, 2000, 2001; Muller and Nisbet, 2000; Nisbet et al., 2004; Rinke and Vijverberg, 2005; Vanoverbeke, 2008), because detailed data on the physiology of Daphnia is available and Daphnia is a key herbivore in freshwater systems. Therefore, we will apply our model concepts to the physiology of Daphnia as model organism and exemplify the implication of the physiological model on predator-prey interactions using the Daphnia-algae system.

Two main classes of DEB-models are commonly distinguished: net assimilation models (NAM) which are sometimes also-called $\kappa$ rule models (e.g. Kooijman, 2000, 2001; Muller and Nisbet, 2000) and net-production models (NPM) (e.g. Noonburg et al., 1998; Lika and Nisbet, 2000; Nisbet et al., 2004). Both types of models have been employed in physiologically structured Daphnia population models: the NAM e.g. by DeRoos et al. (1992), Rinke and Vijverberg (2005) and Schalau et al. (2008) and the NPM e.g. by Nisbet et al. (2004) and Vanoverbeke (2008). The main difference between the two types of individual level models is the allocation of assimilated energy $(A)$ to somatic growth $(G)$, reproduction $(R)$ and maintenance $(M)$ (Fig. 1). $A, G, R$ and $M$ represent fluxes of carbon. In the most simplified form the models can be described by (see also Noonburg et al,, 1998):

NAM : $\quad$ NPM :

$G=\kappa \cdot A-M \quad G=\lambda \cdot(A-M)$

NAM : $\quad$ NPM

$R=(1-\kappa) \cdot A \quad R=(1-\lambda) \cdot(A-M)$

In the NAM-approach a constant fraction $\kappa$ of the assimilated energy is allocated to somatic growth and maintenance and the remaining fraction of the assimilated energy is channelled to reproduction (Fig. 1A). In the NPM-approach maintenance is satisfied first and only the flux of the energy surplus, the net-production energy $P=A-M$, is allocated to reproduction and growth. The fraction of $P$ that goes to somatic growth is $\lambda$ and the remaining energy is allocated to reproduction (Fig. 1C).

The basic NAM and NPM (Eq. (1a) and (1b)) have been extended to include reserves which have been treated in different ways, e.g. in form of an energy compartment which is filled by assimilation and from which energy is distributed to reproduction, maintenance and growth (e.g. Muller and Nisbet, 2000; illustrated in Fig. 1B and D), or in form of body mass that serves to satisfy maintenance under starving conditions (e.g. McCauley et al., 1990b; Gurney et al., 1990; Rinke and Vijverberg, 2005; Vanoverbeke, 2008). In the first case the storage compartment requires a modification of Eq. (1a) and (1b) because storage occurs before energy allocation to growth, reproduction and maintenance and acts as a buffer between influx of energy and the energy flux to physiological properties (Fig. 1B and $\mathrm{D})$. Note, however, that the allocation scheme distributing the energy flux $S$ from the storage compartment to reproduction, growth and maintenance can follow the NAM-approach (Fig. 1B), i.e. channel a fixed fraction of the energy to reproduction and 
Table 1

Physiological state relationships and physiological rates.

\begin{tabular}{|c|c|}
\hline Description & Formula \\
\hline Length-weight relationship & $W_{\text {ideal }}=\gamma \cdot L^{q \mathrm{wr}}$ \\
\hline Critical weight & $W_{\text {crit }}=f_{\text {crit }} \cdot W_{\text {ideal }}$ \\
\hline Maximum fractional weight loss & $f_{c r i t}=a_{f c r i t}+\max \left\{-b_{f c r i t},\left(L-L_{n}\right) \cdot \frac{b_{c r i t}-a_{c r i t}}{c_{f c r i t}-L_{n}}\right\}$ \\
\hline Ingestion rate & $I=p_{\text {ing }} \cdot\left[q_{\lambda} \cdot f_{r}(F) \cdot f_{T}(T)\right.$ \\
\hline Functional response & $f_{F}(F)=\frac{F}{F+k_{F}}$ \\
\hline Temperature scaling & $f_{T}(T)=e^{\left(T_{A} / T_{r f}-T_{A} / T\right)}$ \\
\hline Assimilation rate & $A=I \cdot E_{A}=a_{F, T} \cdot L^{q_{A}}$ \\
\hline Assimilation factor & $a_{F, T}=p_{\text {ing }} \cdot E_{A} \cdot f_{F}(F) \cdot f_{T}(T)$ \\
\hline Assimilation efficiency & $E_{A}=E_{A, \max }-\left(E_{A, \max }-E_{A, \min }\right) \cdot f_{F}(F)$ \\
\hline Maintenance coefficient & $m_{F, T}=\left(m_{F, \text { min }}+\left(m_{F, \max }-m_{F, \text { min }}\right) \cdot f_{F}(F)\right) \cdot f_{T}(T)$ \\
\hline Maintenance & $M=m_{F, T} \cdot W^{q M}$ \\
\hline Scaling factor for the energy allocation if $M / A<1$ & $f_{M \mid A<1}=\frac{1}{2}-\frac{1}{2} \tanh \left(\frac{10}{c} \cdot\left(\frac{W_{\text {ideal }}-W}{W_{\text {ideal }}-W_{\text {crit }}}-c\right)\right)$ \\
\hline Energy allocation factor between somatic mass and reproduction for adults & $\lambda_{\text {adult }}=\frac{1}{1-M / A} \cdot(\kappa-M / A)$ \\
\hline Factor describing energy allocation between somatic growth and reproduction if $M / A<1$ & $\lambda= \begin{cases}\lambda_{j u t} & L<L_{S A M} \\
\max \left(0, \lambda_{\text {adult }}\right) & L \geq L_{S A M}\end{cases}$ \\
\hline \multirow[t]{3}{*}{ Factor describing energy allocation to somatic mass growth and somatic length growth } & $\begin{array}{l}\lambda_{W}=\lambda \cdot f_{M / A<1}+\left(1-f_{M / A<1}\right) M / A<1 \\
\lambda_{W}=1 \quad M / A \geq 1\end{array}$ \\
\hline & $\lambda_{L}=\lambda \cdot f_{M / A<1} M / A<1$ \\
\hline & $\lambda_{L}=0 \quad M / A \geq 1$ \\
\hline Somatic growth rate $\left(W>W_{c r i t}\right)$ & $\frac{d W}{d t}=\lambda_{W} \cdot(A-M) \equiv g_{W}(W, L, F, T)$ \\
\hline Length growth rate $\left(W>W_{\text {crit }}\right)$ & $\frac{d L}{d t}=\lambda_{L} \cdot(A-M) \cdot\left(\frac{d W}{d L}\right)^{-1} \equiv g_{L}(W, L, F, T)$ \\
\hline Reproductive rate $\left(W>W_{\text {crit }}\right)$ & $\frac{d E}{d t}=\left(1-\lambda_{W}\right) \cdot(A-M) \cdot c_{E}^{-1} \equiv p_{E}(W, L, F, T)$ \\
\hline Mortality rate $\left(W>W_{\text {cric }}\right)$ & $r_{m}=d_{b}$ \\
\hline Rates for $W \leq W_{\text {crit }}$ & $g_{W}=g_{L}=p_{E}=0 ; r_{m}=d_{c r i t}$ \\
\hline Egg development time & $D=a_{e g g} \cdot e^{\left.\left.b_{e g g} \cdot \ln (T]^{\circ} \mathrm{C}\right]\right)+c_{e g g} \cdot \ln \left(\left.T\right|^{\circ} \mathrm{C}\right)^{2}}$ \\
\hline Egg loss rate from clutch & $r_{E}=\frac{1}{D}$ \\
\hline
\end{tabular}

(A) NAM

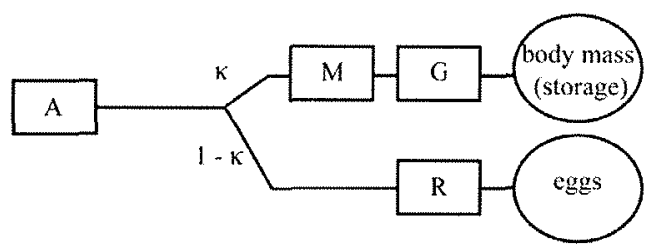

(C) NPM

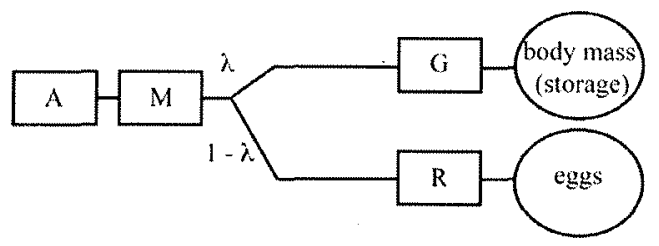

(B) NAM-extended

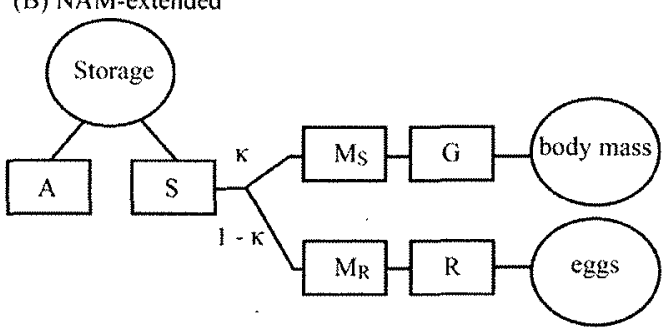

(D) NPM-extended

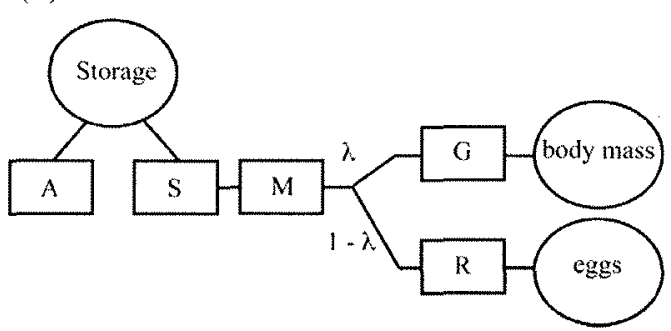

Fig. 1. Schematic representation of different energy allocation models considering assimilation $A$, maintenance $M$, reproduction $R$, growth $G$, and the energy flux from storage $(S)$. Energy fluxes (in terms of carbon) are depicted as squares whereas energy compartments are shown as ellipses. The basic versions of net assimilation model, NAM, (A) and net-production model, NPM, (C) with somatic mass as storage for reserves (NAMs: McCauley et al., 1990b; Gurney et al., 1990; Noonburg et al., 1998; Rinke and Vijverberg, 2005; Schalau et al., 2008; NPMs; Nisbet et al., 2004; Vanoverbeke, 2008). In (B) and (D) these models are extended by an independent storage compartment. The NAM in (B) additionally distinguishes between maturity maintenance $M_{R}$ and somatic maintenance $M_{S}$ (Kooijman, 2000; Muller and Nisbet, 2000). Here, approach (C) is adopted and the allocation factor $\lambda$ is made dependent on the energy flux ratio $M / A$. 
the remaining energy to maintenance and growth, or the NPMapproach (Fig. 1D), i.e. first satisfy maintenance and then allocate the remaining energy between somatic growth and reproduction. In the second concept of reserves, i.e. when body mass acts as reserve that can be mobilized to satisfy maintenance under starving conditions, the storage occurs after the allocation of energy to reproduction and somatic growth and thus Eq. (1) adequately describes NAM and NPM under non-starving conditions. Here, we adopt the latter approach assuming storage in form of body mass and the following discussion of NAM and NPM is derived for this concept. However, the discussion also applies to the allocation of energy flux from the storage compartment.

As a further extension, Kooijman (2000) distinguished between somatic maintenance $M_{S}$ and maturity maintenance $M_{R}$ and assumed that a part of the energy flux to reproduction $R$ is consumed by $M_{R}$ and that only the remaining energy flux $R_{R}$ is available to build eggs or reproductive structure, respectively (Fig. 1B). In NPMs (Noonburg et al., 1998; Nisbet et al., 2004; Vanoverbeke, 2008 ) and in the NAMs of McCauley et al. (1990b), Gurney et al. (1990), Rinke and Vijverberg (2005) and Schalau et al. (2008) maturity maintenance is not considered explicitly (Fig. $1 \mathrm{~A}, \mathrm{C}$ and $\mathrm{D}$ ). Here we adopt the latter approach and assume that maturity maintenance is included in $M$.

Typically, $\kappa$ in NAM is assumed to be constant (Kooijman, 1986, 2000, 2001; Rinke and Vijverberg, 2005). According to McCauley et al. (1990b) in adult Daphnia the proportion of assimilated energy allocated to reproduction $(1-\kappa$ in Eq. (1b)) does not increase with size which also supports a constant value of $\kappa$. In NPM the allocation factor $\lambda$ is commonly assumed to depend on the physiological state of the organism, usually the weight $W$ (Noonburg et al., 1998; Nisbet et al., 2004; Vanoverbeke, 2008). NAM and NPM are often considered controversial concepts. However, the NAM of Eq. (1) is equivalent to the NPM of Eq. (1) if the factor $\lambda$ is assumed to depend on a simple function of the energy fluxes $A$ and $M$ rather than on a function of the physiological state of the organism. From Eq. (1a) follows that a NPM with

$\lambda=\frac{\kappa \cdot A-M}{A-M}=\frac{1}{1-M / A} \cdot\left(\kappa-\frac{M}{A}\right)$

provides exactly the same energy allocation to maintenance, growth and reproduction as the NAM with parameter $\kappa$. The energy allocation factor $\lambda$ can be described as a function of $M / A$ as in Eq. (2), or as a linear function of $A / P: \lambda=1-(1-\kappa) \cdot A / P$ (see Eq. (1b)). In both cases, the NPM model predicts exactly the same temporal development of length, weight and egg production as the corresponding NAM. Note that in Eq. (2) only one parameter is required to describe the energy allocation factor $\lambda$, namely $\kappa$, that also is required in the $\kappa$-rule based NAM.

In addition to the energy allocation scheme, the weight to length relationship and the parameterization of assimilation and maintenance are key factors determining the dynamic behaviour of somatic growth and reproduction. A commonly used representation is (Kooijman, 2000, 2001):

$$
\begin{aligned}
& M(W)=m_{F, T} \cdot W^{q_{M}} \\
& A(L)=a_{F, T} \cdot L^{q_{A}} \\
& W(L)=\gamma \cdot L^{q_{W L}}
\end{aligned}
$$

$W$ represents somatic mass, $L$ length, $\gamma$ is a constant, $m_{F, T}$ and $a_{F, T}$ are functions that depend on food and temperature and $q_{M}, q_{A}$ and $q_{W L}$ are the exponents in the different relationships. Kooijman (1986, 2001) provided arguments for using $q_{M}=1, q_{A}=2$ and for isomorphs $q_{W L}=3$. These exponents were employed in several models of Daphnia by e.g. Muller and Nisbet (2000) and Rinke and Vijverberg (2005). The parameters suggested by Kooijman imply that $M \sim L^{3}$ (Kooijman, 2001). However, other exponents than 3 have also been used in the length to weight relationship for Daphnia (e.g. 2.4 by Nisbet et al., 2004).

The temporal changes in the characteristic properties $W, L$ and the number of eggs per individual (clutch size $E$ ) describing the physiological state of the organism can be calculated from the differential equations:

$$
\begin{aligned}
& \frac{d W}{d t}=\lambda_{W} \cdot(A-M) \\
& \frac{d L}{d t}=\lambda_{L} \cdot(A-M) \cdot\left(\frac{\partial W}{\partial L}\right)^{-1} \\
& \frac{d E}{d t}=\left(1-\lambda_{W}\right) \cdot(A-M) \cdot\left(c_{E}\right)^{-1}-r_{E} \cdot E
\end{aligned}
$$

Eq. (4) are the basic equations of a NPM treating $L$ and $W$ as independent state variables. The energy allocation factors $\lambda_{W}$ and $\lambda_{L}$ are equal for non-starving conditions. The carbon investment per egg, $C_{E}$, and the egg release rate $r_{E}$ are assumed to be constant. The factor $\partial W / \partial L$ depends on the length to weight relationship and is $3 \gamma L^{2}$ when $q_{W L}=3$. Because we assume a fixed weight for eggs and neonates the model cannot consider maternal effects. The latter would require to model egg development and the transformation of energy from the egg to neonate stage (see Kooijman, 2009). Incorporating such a detailed model on egg development in the overall model and its validation with data is beyond the scope of this study and will remain a challenging task in future work.

The behaviour and performance of the individual model developed here will be exemplified using parameter values that are specific for $D$. galeata (see Appendix Table A1). These parameter values will be modified when describing $D$. pulex (see Appendix Table A2).

\subsection{Consequences and conceptual limitations of NPM and NAM}

\subsubsection{Energy allocation factor $\lambda$ in NPM}

In the NAM with a constant $\kappa$ and under constant food levels, length growth ceases when $M=\kappa A \Rightarrow M / A=\kappa$ (see Eq. (1)) and the largest daphnids invest a significant proportion $(1-\kappa)$ of the assimilated energy into reproduction. In contrast to the $\kappa$-rule employed in the NAM, a constant energy allocation factor $\lambda$ in the NPM leads to length growth that continues until $M(L)=A(L)$. Then, the largest organisms not only are significantly heavier and larger than in the NAM, but they also need all assimilated energy for maintenance and thus cannot invest into reproduction. Hence, reasonable applications of the NPM require a dynamic adjustment of $\lambda$.

Noonburg et al. (1998), Nisbet et al. (2004) and Vanoverbeke et al. (2008) assumed that $\lambda$ depends on the physiological state of Daphnia and decreased $\lambda$ with increasing somatic mass. For example, Nisbet et al. (2004) assumed $\lambda \sim 1 /\left(c_{1}+c_{2} \cdot W\right)$ which implies that somatic growth slows down substantially with increasing body mass. However, because $\lambda$ is always greater than 0 , growth still continues until the organism reaches a weight at which all assimilated energy is consumed by maintenance leaving no energy for reproduction. In addition, allocation rules based on the physiological state of an organism imply that at a given body mass or size the fraction of surplus energy allocated to growth is independent of food level. However, animals at same physiological state experiencing different food levels can be expected to adopt different strategies in the allocation of the energy surplus to reproduction and growth because the trade-off between present and future reproduction depends on the total assimilated energy available.

\subsubsection{Decreasing food levels and maturation costs in NAM}

When food levels decrease situations can occur where assimilation is larger than maintenance but maintenance cannot be satisfied if the $\kappa$-rule is applied, i.e. $1 \geq M / A>\kappa$. Under these conditions the application of the $\kappa$-rule would imply that individuals invest 
into reproduction at the cost of starvation. However, according to Kooijman (2001) maintenance is always satisfied first, and hence $A-M$ instead of $(1-\kappa) \cdot A$ is allocated to reproduction if $M / A>\kappa$ (see e.g. Muller and Nisbet, 2000). Conceptually, this implies that the $\kappa$-rule with constant $\kappa$ of the NAM-approach is sacrificed and a netproduction approach is adopted when food levels are decreased sufficiently. Allocation of all net-production energy to reproduction appears to be acceptable for adults but leads to unrealistic situations for juveniles (see below)

In most NAMs immature animals invest into reproduction to satisfy maturation costs required to build structure for the reproductive apparatus (Kooijman, 2001). Because in the NAM-approach with constant $\kappa$ the ratio between assimilated energy and maintenance decreases with decreasing food levels, the ratio between the energy invested into maturation costs and the energy invested into somatic growth is much larger at low than at high food levels. Hence, application of the $\kappa$-rule to juveniles implies that at a given somatic mass, accumulated maturation costs depend on food level. If $\kappa A=M$, the $\kappa$-rule approach and the assumption that maturation costs are investigated entirely into reproductive structure (as e.g. in the NAMs of Rinke and Vijverberg, 2005; Schalau et al., 2008) results in NAMs to the rather unrealistic condition that juveniles invest into reproductive structure at no growth. Note that $\kappa A=M$ is not a starving condition. The above consequence of the $\kappa$-rule could be avoided if a so-called maturity maintenance $M_{R}$ is introduced and is distinguished from somatic maintenance $M_{S}$ (Kooijman, 2000; Muller and Nisbet, 2000; see Fig. 1B), and if the energy allocated to reproduction in juveniles is entirely used for $M_{R}$ if $\kappa A=M_{S}$, i.e. $M_{R}=(1-\kappa) A$, implying $M_{R}=M_{S} \cdot(1-\kappa) / \kappa$. However, when using this relationship for $M_{R}$ also for adults, the NAM with explicit $M_{R}$ and $M_{S}$ can be rearranged into a NPM with $M=M_{R}+M_{S}$ and energy allocation factor $\lambda=\kappa$, implying a constant ratio between somatic mass and egg production. The latter had been rejected by Noonburg et al. (1998) as inconsistent with observations. Note further, that in NAMs also under conditions $K A<M$ and $A>M$ all net-production energy is investigated into maturation costs at no growth. In these cases the $M_{R}$ above would lead to a negative energy flux to reproductive structure.

Although maturation time varies substantially with food concentration, the size at first clutch in daphnids appears to be independent of food concentration (McCauley et al., 1990a: $D$. pulex; Urabe, 1988 and Rinke and Vijverberg, 2005: D. galeata; Kooijman, 2000: Daphnia magna). Assuming that daphnids of same size have the same structural requirements for reproduction, investment into reproductive structure can be expected to be independent of food. This and the discussion above on the difficulties arising from the energy allocation to maturation when $\kappa A \leq M$ and $A>M$ suggests that energy allocation to maturation costs in juveniles may differ from energy allocation to reproduction in adults.

The difficulties arising from the food-dependency of maturation costs become severe in situations when $1 \geq M / A>\kappa$, i.e. when the entire net-production energy acquired by juveniles flows into maturation costs leaving no energy for somatic growth. Investment into maturation however only pays back if the organism reaches the size at maturation and hence, allocation of all net-production energy into maturation costs cannot be adaptive. Therefore, it appears reasonable to assume that non-starving juveniles allocate at least some energy to somatic growth even if $K A \leq M$. Such a modification of the $\kappa$-rule can solve the difficulties that led to the introduction of maturity maintenance.

According to McCauley et al. (1990b) somatic growth of juveniles is faster than would be expected from a Bertalanffy type growth curve that extends from neonates to largest sized adults. This suggests that juveniles invest a larger proportion of assimilated energy into growth than adults, i.e. juveniles invest a smaller proportion of $A$ into maturation costs than adults into reproduction.

\subsection{A new energy flux based NPM}

Our DEB-model employs a net-production allocation scheme in which $\lambda$ is based on energy fluxes rather than on the physiological state of the organism. Thus, the model does not require a switch in allocation scheme under low food conditions and considers not only the physiological state but also the environmental conditions in the energy allocation between somatic growth and reproduction. Since all DEB-models require that $M$ and $A$ are "measured" by the organism, and the energy flux ratio $M / A$ not only provides a good description of the limit of growth $(M / A=\kappa)$ but also can be used to determine the onset of starvation $(M / A=1)$, we base the allocation of net-production energy in our NPM on the energy flux ratio M/A. For non-starving conditions $M / A$ is smaller than 1 and positive.

Several criteria constrain the functional form of $\lambda$ and the specific choice of $\lambda$ adopted in our model is motivated in the following paragraphs.

\subsubsection{Mature organisms under constant food levels}

Under a constant food level mature organisms increase the fraction of net-production energy allocated to reproduction, i.e. they decrease $\lambda$, the larger they get, thus slowing down somatic growth. At some size somatic growth must stop and $\lambda$ should become zero. Because the $\kappa$-rule allocation reasonably well described the life history of adult daphnids at different food levels, we adopt Eq. (2) for $\lambda$ thus integrating the $\kappa$-rule approach into the NPM. According to Eq. (2) $\lambda$ becomes zero when $M / A=\kappa$

Fig. 2 A shows the application of the $\kappa$-rule based NPM ( $\kappa$-rule NPM) to life history data of $D$. galeata using the parameterization of the weight to length relationship, of $M$ and of $A$ by Rinke and Vijverberg (2005) (Table 1 and Appendix Table A1). Because the $\kappa$-rule based NPM provides exactly the same energy fluxes as the corresponding NAM it also predicts the same temporal changes in physiological state of organisms (see results in Rinke and Vijverberg, 2005). Hence, the models cannot be distinguished on the basis of experimental data on physiological state variables or energy fluxes within the organism. Further, in both models the energy allocation scheme requires only one empirical parameter which can be determined from $M / A$ of the largest organisms at constant environmental conditions. Hence, under favourable conditions supporting growth as is the case in laboratory experiments with constant food, there seems to be no advantage of one DEBmodel over the other. Note that the comparison above applies to NAM and NPM as depicted in Fig. $1 \mathrm{~A}$ and $\mathrm{C}$ that do not consider a separate energy storage compartment. If such energy storage is introduced for the $\kappa$-rule NPM and the NAM, as illustrated for the NPM in Fig. 1D, energy fluxes and the development of the state variables are still the same for both models but may differ slightly from the results without the additional energy storage compartment. The NAM of Kooijman (2001) depicted in Fig. 1B may however lead to different results depending on the assumed maturity maintenance.

\subsubsection{Juveniles}

For juvenile organisms we assume that $\lambda$ does not vary with $M / A$ but is constant:

if $L<L_{S A M} \quad \lambda=\lambda_{j u \nu}$

where $L_{S A M}$ is the length at maturity and $\lambda_{j u v}$ is the energy allocation factor for juveniles. This assumption guarantees that the ratio of energy allocated to maturation costs and to somatic growth is independent of food level and juveniles grow even under decreasing food levels as long as $M / A<1$. Note that a constant $\lambda$ also implies that all juveniles with the same weight (or size) have invested exactly the same energy into somatic growth and maturation costs, 
(A) Rinke and Vijverberg (2005)
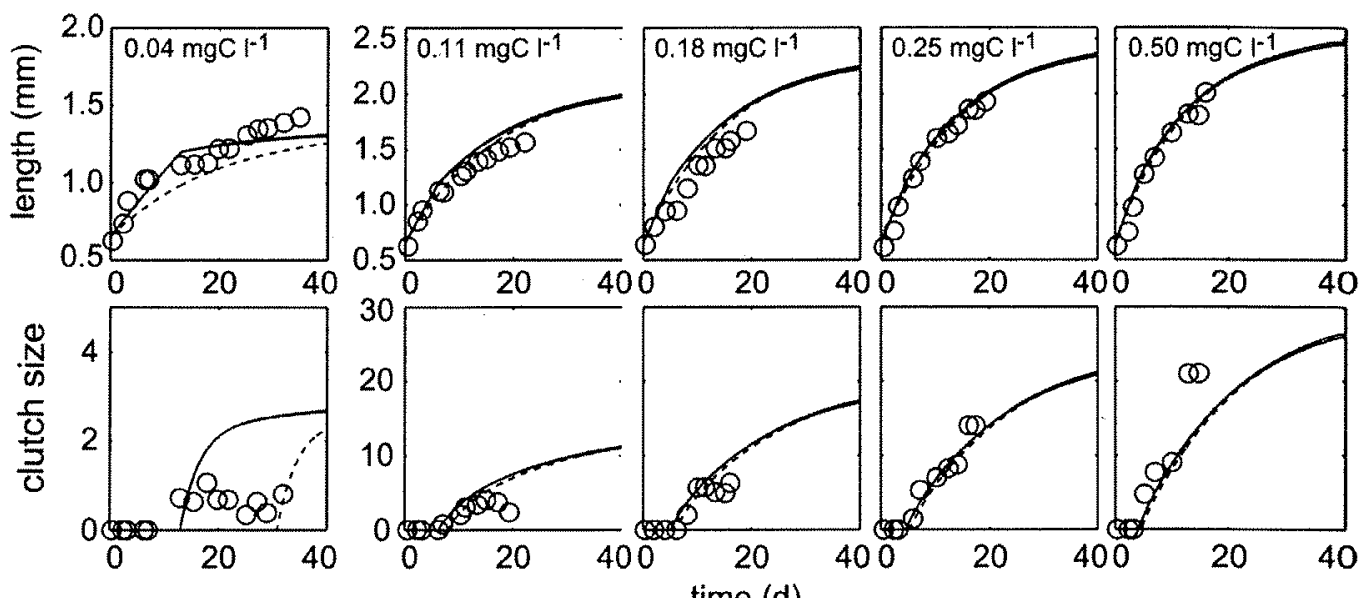

(B) Urabe (1988)

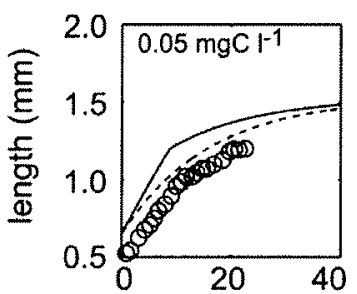

$$
\begin{aligned}
& \text { o data } \\
& \text { - } \text {-rule NPM }{ }_{j u v} \\
& \ldots \text {-. } \text {-rule NPM }
\end{aligned}
$$
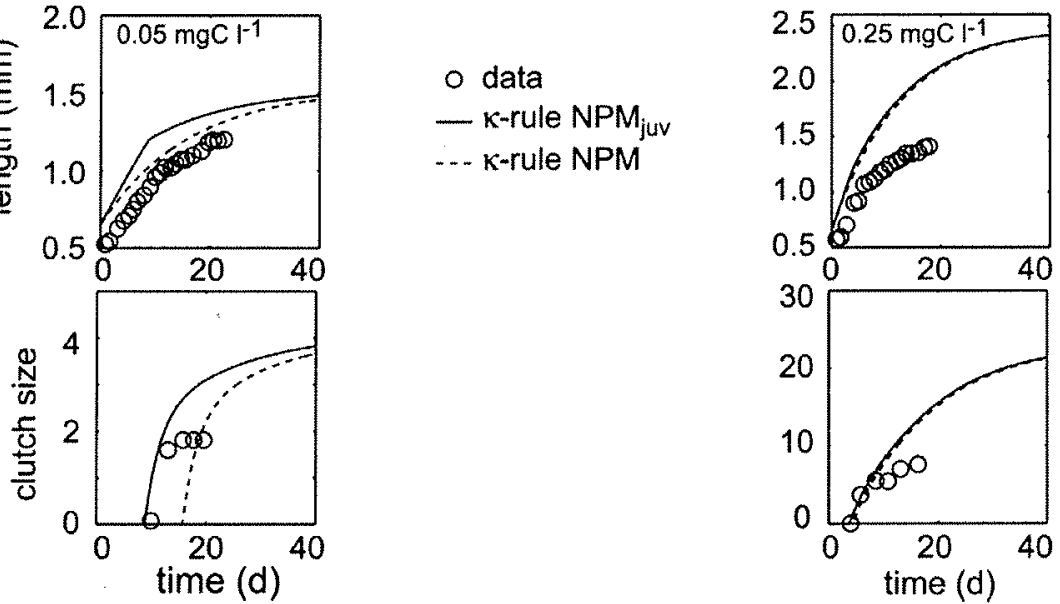

Fig. 2. Comparison of life history observations for Daphnia galeata at different food levels (open circles) with simulations using the new DEB-model, i.e. the $k$-rule based NPM with constant $\lambda$ for juveniles ( $\kappa$-rule NPM Nuv $_{j u}$; solid line), and the $\kappa+$ rule based NPM that employs the $\kappa$-rule also for juveniles ( $\kappa$-rule NPM; dotted line). The latter exactly corresponds to the NAM of Rinke and Vijverberg (2005). (A) Observations on length growth and clutch size of the experiments by Rinke and Vijverberg (2005) and (B) depicts length growth and fecundity in the experiments by Urabe (1988). At the lowest food levels considered in the experiments the models that use $\lambda=\lambda_{\text {juv }}$ predict faster juvenile growth and a significantly shorter maturation time than the model applying the $\kappa$-rule to juveniles. Note that the scale in the panels on the left differ from those of the other panels. Temperature is $17.5^{\circ} \mathrm{C}$ in the simulations of the experiments of Rinke and Vijverberg (2005)(A) and 20 C in the experiments of Urabe (1988) (B). Data are from Rinke and Vijverberg (2005) and from Urabe (1988).

independent of food. However, food conditions affect the growth rate and thus maturation time.

The consequences of the allocation rule for juveniles is exemplified for $D$. galeata (see Fig. 2) for which we have chosen $\lambda_{j u v}=0.29$. This value corresponds to the maximum energy allocation of neonates determined from Eq. (2) at a food level of $0.5 \mathrm{mgCl}^{-1}$, the highest food level considered in Rinke and Vijverberg (2005). Including the energy allocation rule for juveniles (Eq. (5)) in the overall model only slightly alters somatic growth and clutch size of D. galeata at food levels above $0.1 \mathrm{mgCl}^{-1}$ ( $\kappa$-rule $\mathrm{NPM}_{j u v}$, Fig. $2 \mathrm{~A}$ ). At the lowest food concentration, however, the fixed allocation for juveniles in the $\kappa$-rule $\mathrm{NPM}_{j u \nu}$ leads to faster somatic growth of juveniles and to a significant faster maturation (Fig. 2A) than in the $\kappa$-rule NPM. Both effects are in good accordance with observations and improve the agreement between model and data compared to the NAM or the equivalent $\kappa$-rule NPM that applies the $\kappa$-rule also to juveniles. All models including the original NAM of Rinke and Vijverberg (2005) overestimate the clutch size at a food level of $0.04 \mathrm{mgCl}^{-1}$ and $17.5^{\circ} \mathrm{C}$ temperature suggesting that the chosen parameters are not entirely adequate at all food levels. The agreement between simulated and measured clutch size at low food levels could be improved by increasing the carbon content of eggs, i.e. egg size at low food levels (see e.g. Gliwicz and Guisande, 1992; Trubetskova and Lampert, 1995).

Simulations of data on $D$. galeata that were raised at $20^{\circ} \mathrm{C}$ (Urabe, 1988 ) confirm that the new DEB-model, i.e. the $\kappa$-rule $\mathrm{NPM}_{j u v}$, better predicts maturation time in a gradient of food concentrations than a pure NAM (Fig. 2B). Overall agreement between simulations and the data of Urabe (1988) is not as good as for the data of Rinke and Vijverberg (2005) because the latter were the basis for parameter calibration and Daphnia physiology differed between clones (e.g. the size of neonates were smaller in the experiments by Urabe, 1988).

Application of the model to laboratory life history data of $D$. pulex raised under constant food levels demonstrates that only very few parameter adjustments are required to adequately predict somatic growth and reproduction of $D$. pulex (Fig. 3, see Table A2 in Appendix for parameters).

In all model applications the use of a constant $\lambda$ for juveniles results in a faster maturation than would be expected from mod- 

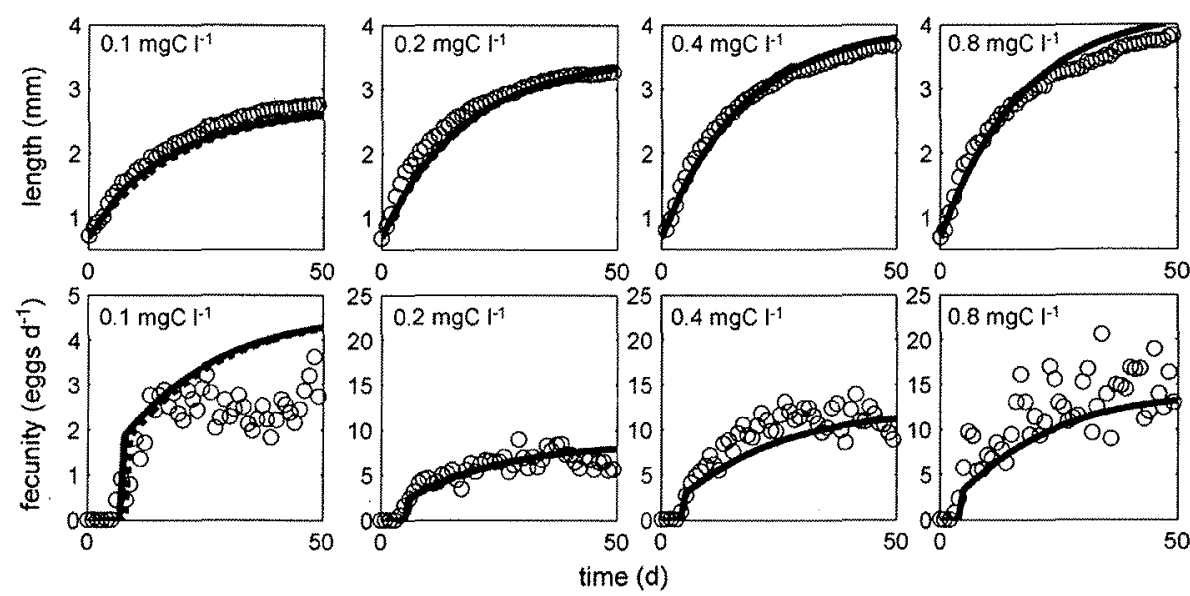

Fig. 3. Comparison of observations (open circles) on length growth and fecundity of Daphnia pulex at different food levels with simulations from the new DEB-model, i.e. the $\kappa$-rule based NPM with constant $\lambda$ for juveniles ( $\kappa$-rule NPM ${ }_{j u y}$, solid line), and the $k$-rule based NPM that uses the $\kappa$-rule also for juveniles ( $\kappa$-rule NPM, dotted line). Data are from Nisbet et al., 2004. The parameters used for Daphnia pulex are listed in Table A2 (Appendix). Temperature is $20^{\circ} \mathrm{C}$.

els that apply the $\kappa$-rule also to juveniles, e.g. NAMs. This effect is especially visible at low food conditions: in the $\kappa$-rule NPM $_{j u v}$ maturation time of Daphnia galeatea is about 18 days shorter at $0.04 \mathrm{mgCl}^{-1}$ and $17.5 \mathrm{C}$ (conditions in the experiments of Rinke \& Vijverberg, 2005) and about 7 days shorter at $0.05 \mathrm{mgCl}^{-1}$ and $20 \mathrm{C}$ (conditions in the experiments of Urabe, 1988) than in the $\kappa$ rule NPM or the equivalent NAM (Fig. 2). In the experiments with $D$. pulex the lowest food levels were rather high such that the advantage of shorter maturation times of the new model ( 1.5 days at $0.1 \mathrm{mgCl}^{-1}$ and $20^{\circ} \mathrm{C}$ ) is not as clear as in the experiments with $D$. galeata. At lower food levels the predicted difference in maturation times between $\kappa$-rule NPM $\mathrm{N}_{j u v}$ and NAM for D. pulex (e.g. 17 days at $0.04 \mathrm{mgCl}^{-1}$ and 20 C) would be similar to that for $D$. galeata. The observations especially on $D$. galeata at low food levels clearly support the shorter maturation times predicted by the $\kappa$-rule $\mathrm{NPM}_{j u v}$ (Fig. 2).

Note, that NAMs could be modified to lead to exactly the same energy allocation in juveniles and adults as in the $\kappa$-rule $N_{P M} M_{j u v}$. In such a NAM energy allocation in adults follows the usual $\kappa$-rule with constant $\kappa$, whereas $\kappa$ is a function of $M / A$ for juveniles, i.e. $k=(1-M / A) \lambda_{j u v}+M / A$ if $L<L_{S A M}$. Using this $k$ in NAMs would resolve the difficulties arising from investment in maturation costs at $\kappa A \leq M$ and $A>M$ without requiring maturity maintenance.

\subsubsection{Decreasing food levels and starvation}

If food levels decrease such that $M / A$ falls within the range $\kappa<M / A<1$ adults are assumed to allocate net-production-energy $P$ to reproduction (as Kooijman, 2000, 2001; Muller and Nisbet, 2000). Hence we have the condition for adults:

if $\kappa<\frac{M}{A}<1 \quad \lambda=0$

which can also be represented by $\lambda=\max (0, \lambda)$ because $\lambda$ of Eq. (2) becomes negative when $M / A>\kappa$. Note that in contrast to adults, juveniles are assumed to allocate the constant fraction $1-\lambda_{\text {juv }}$ of $P$ to reproduction as long as $A>M$.

Under starving conditions ( $M / A>1)$ all assimilated energy is used by maintenance and additional reserves must be mobilized to satisfy maintenance or the organism dies. Two different approaches exist in the literature to describe reserves: Kooijman $(1986,2001)$ and Muller and Nisbet (2000) consider a specific storage compartment in addition to somatic energy and reproductive energy whereas the second approach assumes that assimilated energy is directly distributed to maintenance and production and that body mass acts as a reserve during starving conditions (e.g. McCauley et al., 1990b; Gurney et al., 1990) (see Fig. 1).

Bradley et al. (1991a,b) demonstrated that reproduction ceases under starvation and that death is not immediate even if food levels are set to zero. Further, animals were able to resume growth even after an extended period of starvation (Bradley et al., 1991 a,b). They concluded that allocation of energy to reproduction is immediate and not from an energy storage compartment as suggested by Kooijman (1986). McCauley et al. (1990b) stated that the storage suggested by Kooijman (1986) is too short-lived and that slow reserves stored in body mass are required to support survival of daphnids of typically 8-10 days without algae that was observed by Tessier et al. (1983).

We adopt here the approach by McCauley et al.(1990b) that was also used by Gurney et al. (1990), Rinke and Vijverberg (2005) and Vanoverbeke (2008) and assume that during starvation the energy required for maintenance comes from energy stored in body mass. Starving thus results in weight loss but the size of the individuals remains unchanged (McCauley et al., 1990b). Hence, we have the condition:

$$
\text { if } \quad M / A \geq 1 \quad \begin{gathered}
\lambda_{W}=1 \\
\lambda_{L}=0
\end{gathered}
$$

$\lambda_{W}$ and $\lambda_{L}$ are the allocation factors to weight and length growth, respectively. Under all conditions except if $M / A \geq 1$ and if $W<W_{\text {ideal }}$ (see below), $\lambda_{W}=\lambda$ and $\lambda_{L}=\lambda$. Note, that Eq. (7) also implies that no energy is allocated to reproduction. If $M / A \geq 1$ netproduction energy is negative and the organism looses weight to satisfy maintenance. Therefore, we distinguish between the ideal weight, $W_{\text {ideal }}$, the actual weight $W$, and the critical weight $W_{\text {crit }}$ (see also Vanoverbeke, 2008). $W_{\text {ideal }}$ is calculated from the weight for length relationship (Eq. (3)) and the actual length $L$. $W_{\text {crit }}$ characterizes a point of no return when the organism dies rapidly:

$W_{\text {crit }}=W_{\text {ideal }} \cdot f_{\text {crit }}$

The parameter $f_{c r i t}$, defining the fraction of the ideal weight at which the critical weight is reached, may depend on $L$ (see Table 1 ). According to McCauley et al. (1990b) total losses can reach 50-70\% of body mass.

When organisms reach the critical weight, $W_{\text {crit }}$, the mortality rate $r_{m}$ is assumed to be significantly lager than the background mortality rate. We further assume that daphnids with $W<W_{\text {crit }}$ stop filtering (see Plath, 1998 for adaptive feeding behavior of Daphnia) 
and thus do not assimilate energy:

$$
\text { if } \begin{aligned}
W \leq W_{\text {crit }} & d W / d t=-M \\
& d L / d t=d E / d t=0 \\
& r_{m}=d_{\text {crit }}
\end{aligned}
$$

Note, that in our model the change in weight of daphnids with $W<W_{\text {crit }}$ has no effect on survival of individuals and therefore has no influence on population dynamics. Because daphnids with $W<W_{\text {crit }}$ are assumed to stop filtering they also have no affect on the food resource. However, we demonstrate model sensitivity to this assumption by a simulation in which daphnids with $W<W_{\text {crit }}$ are assumed to remove food at the same rate as unstarved daphnids of the same length.

\subsubsection{Recovery of under-nourished animals}

When food levels become sufficient that $A>M$, under-nourished animals $\left(W_{\text {crit }}<W<=W_{\text {ideal }}\right)$ can recover (e.g. Bradley et al., 1991a,b; Tessier et al., 1983). Energy allocation during recovery from starvation is not very well studied and data on weight accumulation during this phase are not available. Maintenance is given priority over somatic growth and reproduction (e.g. Bradley et al., 1991b; Polishchuk and Vijverberg, 2005) but the distribution of energy flux between the latter two remains uncertain. We adopt here the assumption that the restoration of weight has priority before length growth and allocation to reproduction resumes (e.g. Gurney et al., 1990; Nisbet et al., 2004; Rinke and Vijverberg, 2005; Vanoverbeke, 2008). However, we smooth the transition to ideal weight conditions to avoid an abrupt change in energy allocation and to make the continuous-time model numerically more tractable. Hence, if $M / A<1$ the allocation factors are modified:

$$
\text { if } \begin{aligned}
M / A<1 & \lambda w=\lambda \cdot f_{M / A<1}+\left(1-f_{M / A<1}\right) \\
& \lambda_{L}=\lambda \cdot f_{M / A<1} \\
& \text { with } f_{M / A<1}=\frac{1}{2}-\frac{1}{2} \tanh \left(\frac{10}{c_{M / A<1}} \cdot\left(\frac{W_{\text {ideal }}-W}{W_{\text {ideat }}-W_{\text {crit }}}-c_{M / A<1}\right)\right)
\end{aligned}
$$

The scaling factor $f_{M / A<1}$ is very close to 0 at $W_{\text {crit }}$ and 1 at $W_{\text {ideal }}$. The parameter $c_{F M / A<1}$ defines the weight at which $f_{M / A<1}=0.5$, i.e. $W=W_{\text {ideal }}-c_{P M / A<1} \cdot\left(W_{\text {ideal }}-W_{\text {crit }}\right)$. Here we chose $c_{f M / A<1}=0.01$. Eq. (10) guarantees that under-nourished daphnids invest net-production energy predominantly to increase body mass without a significant change in length, i.e. $\lambda_{W} \approx 1$ and $\lambda_{L} \approx 0$ if $W<W_{\text {ideal }}-2 c_{f M / A<1} \cdot\left(W_{\text {ideal }}-W_{\text {crit }}\right)$. If $W=W_{\text {ideal }}$ the energy allocation factors $\lambda_{W}$ and $\lambda_{L}$ in Eq. (10) deviate by less than $2.1 \times 10^{-7} \%$ from $\lambda$ (Eqs. (2), (5)-(7)).

\subsection{Implications of the individual model}

\subsubsection{Length growth and body size}

The maximum length $L_{\max }$ approached asymptotically by adult Daphnia at a given food concentration can be calculated from the equation:

$$
\frac{M\left(L_{\max }\right)}{A\left(L_{\max }\right)}=\kappa \Rightarrow L_{\max }=\left(\kappa \cdot \frac{a_{F, T}}{m_{F, T} \cdot \gamma}\right)^{1 /\left(q_{M} \cdot q_{W L}-q_{A}\right)}
$$

Assuming that the exponents in Eq. (3) correspond to those suggested by Kooijman (2001): $q_{W L}=3, q_{A}=2, q_{M}=1$, length growth of juveniles $\left(\lambda=\lambda_{j u v}\right)$ follows Bertalanffy growth (see Appendix). In our model also adults follow Bertalanffy type length growth. However, the adults grow at a slower rate than juveniles with $\lambda=\lambda_{\text {juv }}$. The analytical solution of length growth (see Appendix) is only valid for specific sets of exponents describing the length dependence of maintenance, assimilation and length to weight relationship, i.e. Bertalanffy type solutions for length growth of juveniles and adults follow if $q_{W L}=q_{A}-1$ and $q_{M}=1$. Note that the analytical solutions of length growth assume constant food conditions.

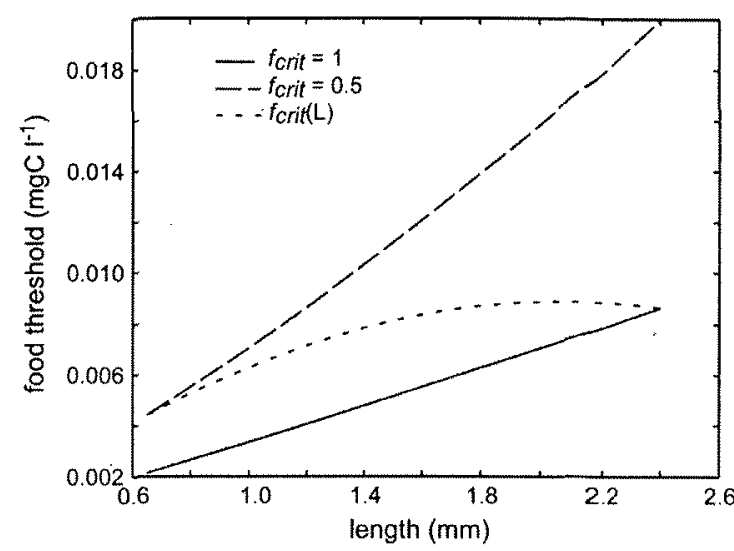

Fig. 4. Critical food level for Daphnia as function of length for different $f_{\text {crit }}$

Critical food levels for Daphnia increase with increasing length. They are significantly reduced if the critical weight for survival is substantially smaller than the ideal weight. $f_{\text {crit }}(L)$ (Table 1, Table A1) increases linearly from $f_{\text {crit }}=0.999$ at $L=0.65 \mathrm{~mm}$ to $f_{\text {crit }}=0.5$ at $L=2.4 \mathrm{~mm}$.

\subsubsection{Starvation and critical food level}

According to Eq. (3), the ratio $M / A$ can be written as function of length:

$\frac{M}{A}=\frac{m_{F, T}}{\alpha_{F, T}} \gamma^{q_{M} L^{\left(q_{W L} \cdot q_{M}-q_{A}\right)}}$

where $m_{F T}, a_{F T}, \gamma$ are positive constants and $q_{W L}, q_{M}$ and $q_{A}$ are the exponents in the weight for length relation ship, the weight dependence of maintenance and the length dependence of assimilation, respectively. Because in all DEB-models of daphnids we know of $q_{W L} \cdot q_{M}$ is larger than $q_{A}$, the ratio $M / A$ typically increases monotonically with $L$ (e.g. $M / A$ increases linearly with $L$ if the exponents $q_{W L}=3, q_{A}=2, q_{M}=1$ of Kooijman, 2001 are used). Consequently, the larger the organism the higher is the food concentration required to satisfy maintenance. Thus, large organisms begin to starve (i.e. to loose weight) at a higher food level than small organisms. Apparently, although the food level at which starvation sets in decreases with increasing size for different species (Gliwicz, 1990), within one species, physiological rates suggest an increase in the food level at starvation with increasing body size. The latter also implies that in a size structured population exposed to slowly decreasing food levels, large animals suffer first from starvation and the smallest animals last. As a consequence, large organisms typically require a higher food level for survival, which however also depends on the size dependence of the tolerable weight loss (see Fig. 4).

In the approach taken here, weight loss reduces maintenance but assimilation remains at a high level because the animal's size, which determines assimilation, is assumed to remain unchanged under starving conditions (e.g. McCauley et al., 1990b; Vanoverbeke, 2008). Hence, the ratio of $M / A$ becomes smaller under starving conditions. This concept makes possible that large organisms can survive very low food levels for extended periods of time. Even a new steady state can be reached under starving conditions at which underweight organisms can satisfy maintenance completely. The critical food level for survival then depends on the maintenance at the critical weight and the assimilated energy at the length of the corresponding ideal weight (see Fig. 4). Therefore, the food level at which the critical weight is reached is lower than the food level at which starvation sets in.

If food is reduced very rapidly to zero as in the experiments of Tessier et al. (1983) or Bradley et al. (1991b) storage causes a delay in death because of dynamic reasons. At zero food $A=0$ and $\lambda_{W}=1$ the change in weight can be calculated from $d W / d t=-M=-m_{F=0, T} \cdot W^{q_{M}}$ (Eqs. (3) and (4)). Using $q_{M}=1$ and 


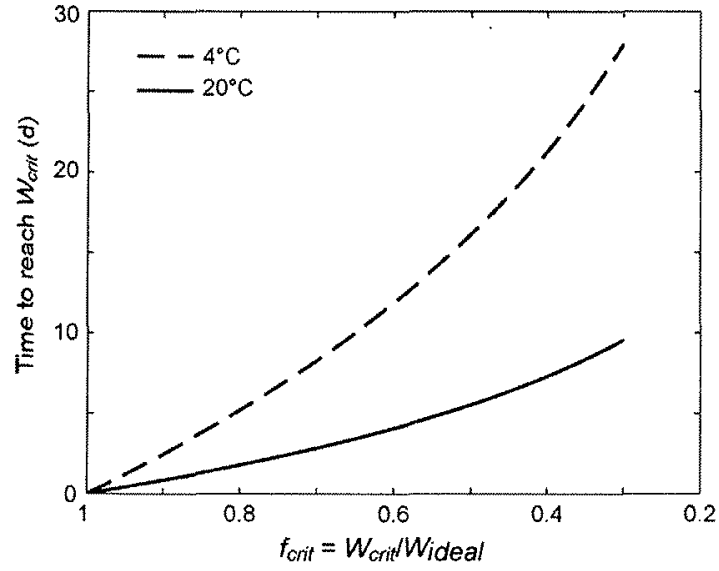

Fig. 5. Time to reach the critical weight at zero food for Daphnia $\left(t_{\text {crit }}\right)$ as function of $f_{\text {crit }} . t_{\text {rrit }}$ increases with increasing $f_{\text {crit }}$ independent of the initial weight and thus also the size of the organism. At higher temperatures $t_{c r i t}$ is smaller than at low temperatures because maintenance requirements increase with temperature.

the parameterization in Table 1 weight decreases exponentially $W(t)=W_{\text {ideal }} \cdot e^{-\left(m_{F_{\text {min }}} f_{f_{T}}(T)\right) \cdot t}$. The time $t_{\text {crit }}$ required for the weight loss from $W_{\text {ideal }}$ to $W_{\text {crit }}$ at zero food is $t_{\text {crit }}=-\ln \left(f_{\text {crit }}\right) /\left(m_{F, \min } \cdot f_{T}(T)\right)$. Fig. 5 shows $t_{\text {crit }}$ at different levels of $f_{c r i t}$ and at 2 temperatures.

Under field conditions, food levels usually do not become zero. The change in length, weight, clutch size and abundance of $D$. galeata that was raised at $0.5 \mathrm{mgCl}^{-1}$ and experiences a sudden decrease in food level to values of $0.01 \mathrm{mgCl}^{-1}, 0.005 \mathrm{mgCl}^{-1}$ and $0 \mathrm{mgCl}^{-1}$ is illustrated in Fig. $6 \mathrm{~A}, \mathrm{~B}$, and $\mathrm{C}$ respectively. These calculations are based on the parameters of Table A1 (see Appendix) and assume $f_{\text {crit }}=0.5$. If food levels drop to $0.01 \mathrm{mgCl}^{-1}$ weight of large Daphnia decreases whereas that of small Daphnia increases (Fig. 6A). However, Daphnia at all length reach an equilibrium state with somatic mass above $W_{\text {crit }}$. If food levels drop to $0.005 \mathrm{mgCl}^{-1}$ the weight of large daphnids decreases faster to values below $W_{\text {crit }}$ than that of small daphnids (Fig. 6B). Hence, large daphnids die earlier than small daphnids (Fig. 6B). At zero food all daphnids reach $W_{c r i t}$ at the same time and thus experience the increased death rate at the same time (Fig. $6 \mathrm{C}$ ). Using the same conditions as in Fig. $6 \mathrm{C}$ but assuming $\mathrm{f}_{\text {crit }}=0.999$ instead of $\mathrm{f}_{\text {crit }}=0.5$ results in a faster decline of Daphnia abundance (Fig. 6D). Note, that if $f_{c r i t}$ depends on length, the time to reach $W_{\text {crit }}$ under no-food conditions also is length dependent (Fig. 6E)

\subsubsection{Effect of temperature}

The parameterization of the physiological rates adopted here from Rinke and Vijverberg (2005) assumes that temperature effects scale maintenance in the same manner as assimilation (Table 1) The functional form of $M$ and $A$ can be written as $M(T, F)=f_{T}(T) \cdot M_{F}(F)$ and $A(T, F)=f_{T}(T) \cdot A_{F}(F)$, where $f_{T}(T)$ is the temperature $(T)$ scaling function and $M_{F}(F)$ and $A_{F}(F)$ are the maintenance and assimilation at a reference temperature at different food levels $(F)$, respectively. According to the parameterization above, the energy flux ratio $M / A=M_{F} / A_{F}$ is independent of temperature and thus also the energy allocation $\lambda$ between growth and reproduction (see Eq. (2)). As a consequence, the ratio of somatic mass to cumulative mass in reproduction also does not depend on temperature. Further, temperature has no influence on the maximum length at given food level, the minimum food level before onset of starvation and the critical food level.

However, although the energy allocation between growth and reproduction does not depend on temperature, net-production scales with $f(T): P(F, T)=f(T) \cdot\left(A_{F}(F)-M_{F}(F)\right)$ and therefore depends on $T$. Hence, somatic growth rates and reproduction and also the timing of maturation and overall population growth are strongly affected by temperature. Because $f(T)$ increases with temperature somatic growth is faster in warm waters than in cold waters. Therefore, also the demography of the population depends on temperature, i.e. in the stable age distribution at positive growth the number of individuals decreases stronger with age at high temperatures than at low temperatures. Note also, that at low temperatures the loss rate of body mass during starvation is slower than at high

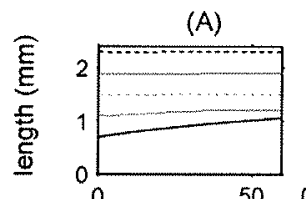

(B)
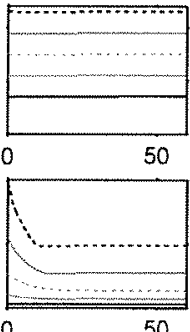

(C)

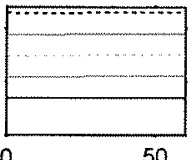

(D)

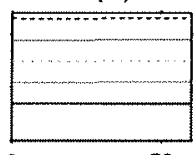

(E)

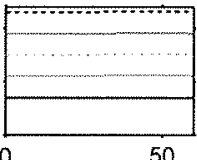

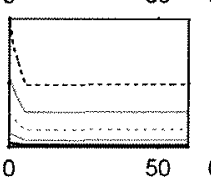
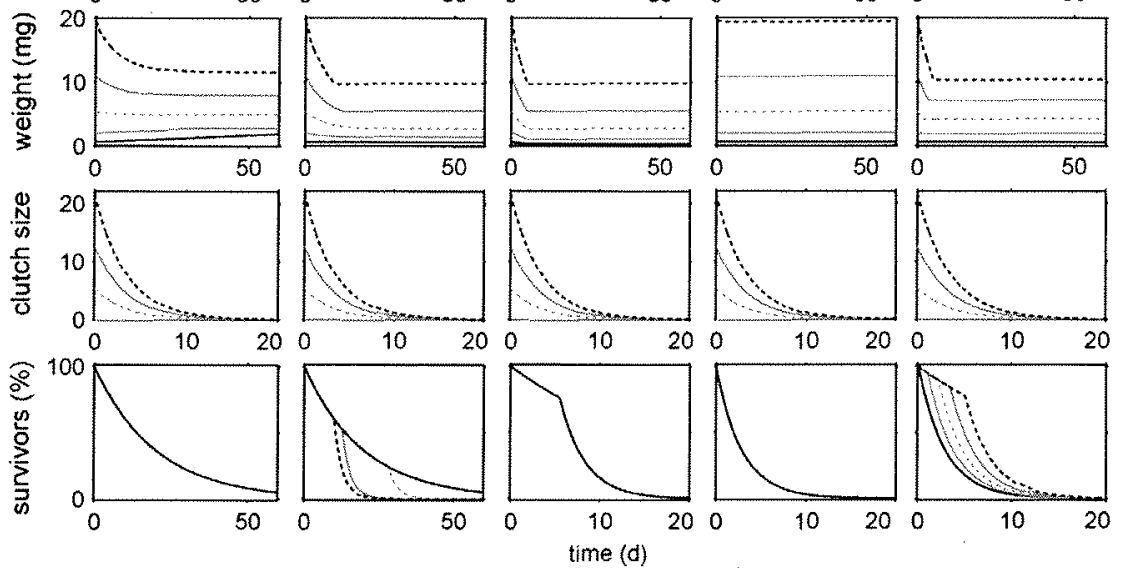

Fig. 6. Impact of reduced food levels on life history properties of Daphnia galeata of different initial size and for different $f_{\mathrm{crit}}$. Daphnia were raised at $0.5 \mathrm{mgCl}{ }^{-1}$ and a temperature of $175^{\circ} \mathrm{C}$ and then suddenly exposed to significantly lower food levels: $0.01 \mathrm{mgCl}^{-1}, 0.005 \mathrm{mgCl}-1,0 \mathrm{mgCl}^{-1}, 0 \mathrm{mgCl}^{-1}$ and $0 \mathrm{mgCl}^{-1} \mathrm{from} \mathrm{left} \mathrm{to} \mathrm{right} \mathrm{column}$ (A-E). The simulations in (A-C) assume $f_{\text {crit }}=0.5$ whereas in (D) $f_{\text {crit }}=0.999$ and in (E) $f_{\text {crit }}(L)$ (see Table 1 and Table A1) increases linearly from $f_{\text {crit }}=0.999$ at $L=0.65$ to $f_{\text {crit }}=0.5$ at $L=2.4 \mathrm{~mm}$. Each line in the plots represents a different sized individual that is exposed to the decreased food level. The solid black line representing the development of daphnids initially with $L=0.7 \mathrm{~mm}$ cover the other lines in the figures demonstrating the fraction of surviving individuals. 
temperatures thus allowing daphnids to survive longer at the low food levels in the cold waters during the winter period.

\section{Implications of the new DEB-model at population level}

\subsection{Incorporation of the new DEB-model into a structured population model}

The implications of individual physiology on population dynamics were investigated by incorporating the individual model into a structured population model (e.g. DeRoos et al., 1992; Rinke and Vijverberg, 2005; Vanoverbeke, 2008). We use the box-car elevator technique (DeRoos et al., 1992) to numerically solve the population development. The box-elevator technique is a Lagrange method to solve partial differential equations by discretizing the population into age cohorts. For each cohort the differential equations describing the change in somatic weight, somatic length, egg production (Eq. (4)) and in addition the change in the number of individuals $N$ and in the mean age, $a$, of the cohort:

$$
\begin{aligned}
& \frac{d N}{d t}=-r_{m}(W) \cdot N \\
& \frac{d a}{d t}=1
\end{aligned}
$$

are solved numerically. $r_{m}$ is the specific mortality rate.

Reproduction requires the generation of new cohorts. After discrete time steps a new first cohort, the cohort in creation, is re-generated and all other cohorts are renumbered. In the renumbering process the cohort with the oldest individuals is usually removed from the population implying sudden death. The number of cohorts and the maximum lifetime of the daphnids determine the time span between subsequent reordering and thus the maximum residence time of daphnids in the cohort in creation.

Because neonates are continuously released into the cohort in creation, the average properties of the daphnids in the cohort in creation are a mixture of the properties of the neonates and of the daphnids already present in this cohort. This mixing process can be accounted for by using the state variables "total biomass" $\left(W_{c}=N_{0} \cdot W_{0}\right)$, "total equivalent length" $\left(L_{\mathcal{C}}=N_{0} \cdot \gamma \cdot\left(L_{0}\right)^{q_{W L}}\right)$ and "total age" $\left(a_{\mathrm{c}}=N_{0} \cdot a_{0}\right)$ instead of mean weight, length and age, respectively:

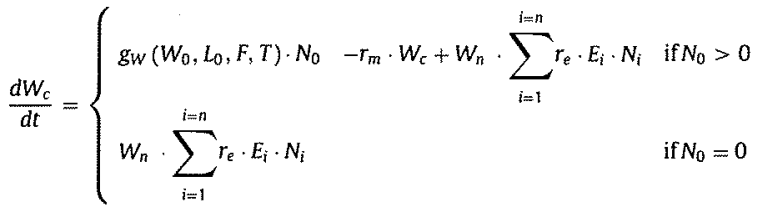

$$
\frac{d L_{c}}{d t}= \begin{cases}\gamma \cdot q_{W L} \cdot L_{0}^{\left(q_{W L}-1\right)} \cdot g_{L}\left(W_{0}, L_{0}, F, T\right) \cdot N_{0}-r_{m} \cdot L_{c} & \\ +W_{n} \cdot \sum_{\substack{i=1 \\ i=n}}^{i=n} r_{e} \cdot E_{i} \cdot N_{i} & \text { if } N_{0}>0 \\ W_{n} \cdot \sum_{i=1} r_{e} \cdot E_{i} \cdot N_{i} & \text { if } N_{0}=0\end{cases}
$$

$\frac{d a_{c}}{d t}=N_{0}-r_{m} \cdot a_{c}$

with $W_{0}=W_{c} / N_{0}$ and $L_{0}=\left(L_{c} /\left(N_{0} \cdot \gamma\right)\right)^{1 / q_{w l}}$.

Note, that the state variables and the differential equations employed here for the cohort in creation differ from those proposed by DeRoos et al. (1992). Incorporation of starvation in a continuoustime frame requires an independent treatment of weight and length in the cohort of creation and the functional form of the state variable $L_{c}$ is needed to guarantee that under non-starving conditions the
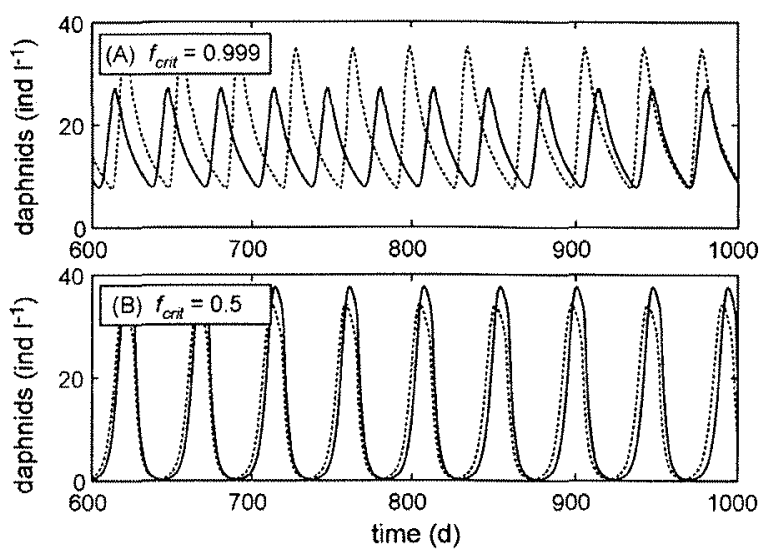

Fig. 7. Cycles in Daphnia abundance in Daphnia-algae interactions assuming different allocation schemes for juvenile daphnids: constant $\lambda_{j u v}\left(\kappa\right.$-rule $\mathrm{NPM}_{j t v}$, solid lines) and $\kappa$-rule for juveniles ( $\kappa$-rule NPM, dotted lines). In the upper panel $f_{\text {crit }}=0.999$ and in the lower panel $f_{\text {crit }}=0.5$. Algae are simulated using a growth rate $a=0.5 \mathrm{~d}^{-1}$ and a carrying capacity of algae $K=0.2 \mathrm{mgCl}^{-1}$. Temperature is $20^{\circ} \mathrm{C}$ in all simulations.

ideal length to weight relationship (see Eq. (3)) holds for the mean mass and the mean length of the daphnids in the cohort in creation. Clutch size is not considered as state variable in the cohort in creation because the fast renumbering $(2 \mathrm{~h})$ prohibits growth to the size at maturity thus making reproduction in the cohort of creation impossible.

The dynamics of food algae is simulated by assuming logistic growth and loss due to herbivory by Daphnia. The loss term is given by the total ingestion of all daphnids from all cohorts (see Table A3 in Appendix

\subsection{Numerical implementation}

Individual and population model are both continuous-in-time models that are numerically integrated using a higher order explicit Runge-Kutta algorithm with dynamic time stepping. The models have been implemented in MATLAB using the ode 45 solver. Time stepping is controlled assuming absolute and relative tolerance levels of $10^{-6}$. In the population model the Runge-Kutta algorithm was applied for the time periods between reordering. The solution of the integration prior to the reordering step was used to generate the start vector for the time period after reordering. We consider 840 cohorts with a life-span of 70 days thus requiring reordering every $2 \mathrm{~h}$. As initial conditions a stable age distribution and the corresponding weight, age and clutch sizes were calculated from an iterative simulation with a total duration of 1000 days assuming constant food conditions. This stable age distribution was normalized to have a total of 1 individual per litre.

\subsection{Impact of physiology under starving conditions on predator-prey cycles}

The dynamic interaction between an age-resolved predator population consisting of $D$. galeat $a$ and the biomass of algal prey at 20 C is illustrated in Figs. 7-9 using the physiologically structured population model that includes the $\kappa$-rule based NPM and employs the parameterization of the physiology of $D$. galeata (Table 1 and Tables A1 and A3 in Appendix).

Fig. 7 compares the consequences of the treatment of juveniles in the individual model, i.e. whether the $\kappa$-rule or a constant allocation $\lambda=\lambda_{j u v}$ is applied for juveniles. Algae growth rate is $\alpha=0.5 \mathrm{~d}^{-1}$ and carrying capacity is $K=0.2 \mathrm{mgCl}^{-1}$. In Fig. 7A the daphnids are assumed to have essentially no reserves $f_{\text {crit }}=0.999$, whereas in 

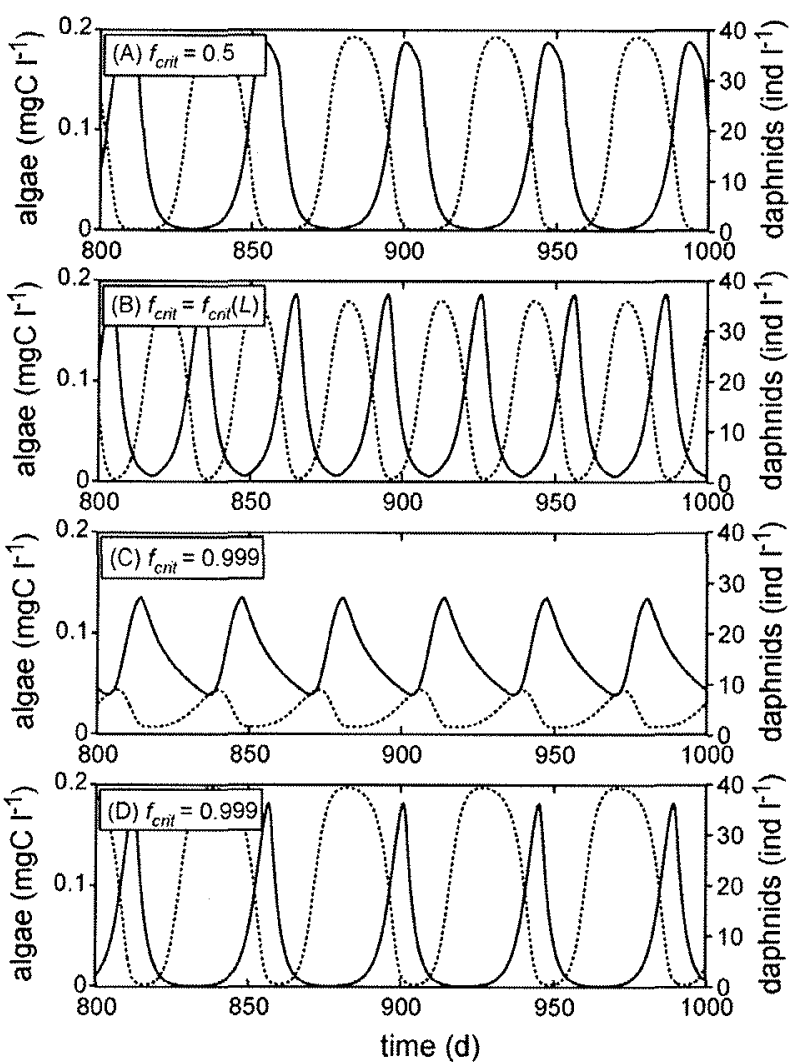

Fig. 8. Predator-prey cycles in Daphnia-algae interactions assuming different physiologies of daphnids under starvation conditions. Amplitudes and periods of the cycles of Daphnia abundance (solid lines) and algae concentration (dashed lines) significantly depend on Daphnia physiology under starving conditions. All simulations are based on the same population model, the same DEB-model ( $k$-rule NPM ${ }_{j u v}$ ), the same parameters for daphnids and the same parameterization of the prey. The only difference between simulations presented in (A), (B) and (C) is $f_{\text {crit }}$ in $(A) f_{\text {crit }}=0.5$, in (B) $f_{\text {crit }}(L)$ (Table 1; Table A1) increases linearly from $f_{\text {crit }}=0.999$ at $L=0.65$ to $f_{\text {crit }}=0.5$ at $L=2.4 \mathrm{~mm}$, and in (C) $f_{\text {crit }}=0.999$. In (C) and (D) the same $f_{\text {crit }}$ is used but the treatment of daphnids with $W<W_{\text {crit }}$ is different. In (D) these daphnids ingest at the same rate as well fed daphnids whereas in (C) these daphnids do not ingest. Carrying capacity is $K=0.2 \mathrm{mgCl}^{-1}$ and temperature is $20^{\circ} \mathrm{C}$ in all simulations.

Fig. $7 \mathrm{~B}$ the daphnids are allowed to loose $50 \%$ of their weight before they die of starvation, i.e. $f_{c r i t}=0.5$. The simulated predator-prey abundances show typical predator-prey cycles. At higher starving potential the predator-prey cycles have longer periods and larger amplitudes than at low starving potential. At high starving potential constant energy allocation in juveniles leads to larger amplitudes than $\kappa$-rule allocation in juveniles. At low starving potential the situation is opposite.

Different values of $f_{\text {crit }}$ not only lead to different periods of the predator-prey cycles but also substantially change the phase relationship between predator and prey peak abundance (Fig. 8A-C). Further, assuming essentially no potential for weight loss $\left(f_{\text {crit }}=0.999\right.$, Fig. $\left.8 \mathrm{C}\right)$ results in much smaller amplitudes of the predator-prey cycles than assuming that the daphnids survive $50 \%$ weight loss $\left(f_{\text {crit }}=0.5\right.$, Fig. 8A). In addition to the level of $W_{\text {crit }}$, also the behaviour of daphnids with weight below $W_{\text {crit }}$ has a strong impact on the predator-prey interaction. Assuming the extreme that daphnids with $W<W_{\text {crit }}$ ingest food at the same rate as well nourished daphnids (Fig. 8D) predator-prey cycles have a substantially longer period and larger amplitudes than in simulations assuming that daphnids with $W<W_{\text {crit }}$ do not ingest food (Fig. 8C).
The simulations suggest that the consequences of a change in the carrying capacity on stage-structured Daphnia-algae interactions significantly depend on the assumptions on the starving potential of daphnids (Fig. 9). Increasing the carrying capacity from $0.2 \mathrm{mgCl}^{-1}$ to $0.5 \mathrm{mgCl}^{-1}$ leads to a substantial increase in the amplitudes of the Daphnia oscillations in the predator and prey

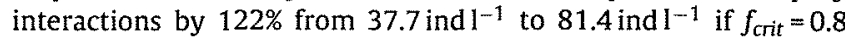
(Fig. 9B and D), whereas the amplitude of the oscillations increases

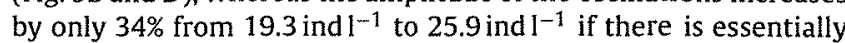
no potential for survivable weight loss $\left(f_{\text {crit }}=0.999\right.$, Fig. 9A and C).

The bifurcation diagrams in Fig. 10 confirm that the amplitudes of the predator-prey limit cycles increase with carrying capacity and decrease with increasing $f_{\text {crit. }}$. The abundances in the bifurcation diagram are calculated from the maximum and minimum abundances of the last 400 days of simulations extending over 8000 days for carrying capacities between $0.05 \mathrm{mgCl}^{-1}$ to $0.085 \mathrm{mgCl}^{-1}$ and extending over 1000 days for all other carrying capacities.

At low carrying capacities below the bifurcation point the system has a stable equilibrium. The Daphnia equilibrium abundance increases with carrying capacity whereas the algae equilibrium concentration is $0.0172 \mathrm{mgCl}^{-1}$ independent of the carrying capacity (Fig. 10B and D black line; blue line is identical and covered). At the algae concentration of $0.0172 \mathrm{mgCl}^{-1}$ the total fertility rate of Daphnia equals their replacement rate. According to the model daphnids reach their size at maturity at this food level within about 30 days and invest all net-production energy to reproduction during the remaining time of their life-span of 70 days. This investment into reproduction lead to sufficient offspring to compensates for the overall losses due to mortality.

At carrying capacities above the range of stable equilibrium, the minimum algae concentration within the limit cycles decreases with increasing carrying capacity. Eventually, the minimum algae concentration becomes so low that daphnids starve, their body weight falls below $W_{\text {crit }}$ and mortality increases substantially. As a consequence, the amplitude of the limit cycles becomes significantly larger. The carrying capacity at which the transition to larger amplitudes occurs increases with decreasing $f_{\text {crit }}$ (see Fig. 10B).

If instead of the constant energy allocation the $\kappa$-rule energy allocation is applied to juveniles, the range of algal carrying capacities at which stable equilibrium occurs becomes very small (Fig. 10B and $D$, red line; green line is identical and covered) and the algae equilibrium concentration is $0.0350 \mathrm{mgCl}^{-1}$, i.e. substantially larger than the $0.0172 \mathrm{mgCl}^{-1}$ mentioned above. In the model applying the $\kappa$-rule to juveniles, daphnids become mature if assimilation is at least $(1-\kappa) \cdot M$ at the size of maturity, whereas in the model with constant energy allocation only an assimilation of $M$ is required. The minimum algae concentration necessary for maturation is $0.034 \mathrm{mgCl}^{-1}$ and $0.011 \mathrm{mgCl}^{-1}$ in $\kappa$-rule and constant energy allocation model for juveniles, respectively. Because reproduction within the life-span must compensate mortality losses including those of the offspring generations the algae equilibrium concentrations are slightly above the minimum algae concentrations required for maturation. If the carrying capacity is below the algae equilibrium concentration, i.e. below $0.0350 \mathrm{mgCl}^{-1}$ in the model assuming the $k$-rule allocation for juveniles, the Daphnia population dies out (Fig. 10D, red line). Consequently, at low algae carrying capacities, daphnids that always invest a constant fraction of net-production energy into growth have a competitive advantage compared to daphnids that strictly follow the $\kappa$-rule (Fig. 10D, black line).

\section{Discussion}

A new DEB-model combining net-production and net assimilation energy allocation concept was developed to allow for an 

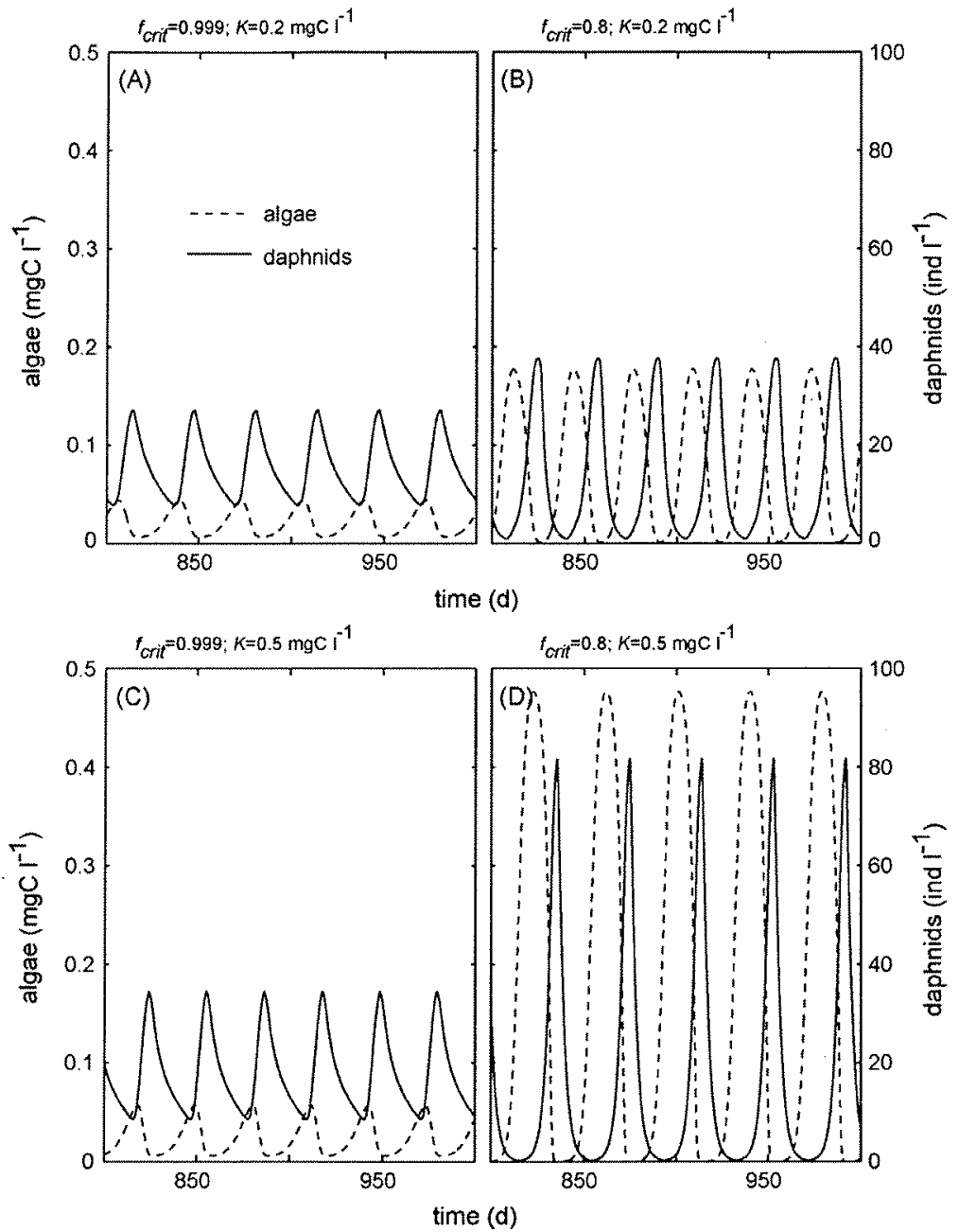

Fig. 9. The effect of prey-carrying capacity on predator-prey cycles assuming different physiologies under starving conditions. An increase in the carrying capacity of the prey has a much smaller effect on the periods and the amplitudes of predator-prey cycles at low starving potential $\left(f_{\text {crit }}=0.999:\right.$ A and $\left.C\right)$ than at high starving potential $\left(f_{\text {crir }}=0.8: B\right.$ and D). Upper panels $(A, B)$ are for carrying capacity $K=0.2 \mathrm{mgCl}^{-1}$, whereas in lower panels $(C, D) K=0.5 \mathrm{mgCl}^{-1}$. Temperature is 20 in all simulations.

improved incorporation of physiology under low food and starving conditions. We demonstrated that net-production and net assimilation models can be designed such that both types of DEB-models provide the same physiological rates and thus also the same temporal development of physiological state properties (length, weight etc.). Hence, the choice of the type of energy allocation approach, NAM or NPM, is not crucial for the adequate description of individual physiology. The net-production allocation scheme and the model formulation adopted in the new DEB-model allow incorporation of physiology under starvation and of different allocation schemes for juvenile growth without requiring a change of the underlying energy allocation concept. Hence, in combination with the structured population model adjusted to consider length and weight as independent state variables the new DEB-model provides an excellent tool to investigate the consequences of low food and starving conditions for population dynamics in predator-prey interactions.

The new individual model adequately describes observations from two different life history studies of $D$. galeata and one study from $D$. pulex that were performed under constant food levels (Fig. 2). However, the numerical experiments with the structured population model demonstrate that population dynamics is not well constrained by the individual physiology based on constant food experiments (Figs. 7-10). Apparently, the starving phase in the predator-prey cycle depending on the physiology under low food and decreasing food levels that is typically not considered in life history experiments significantly affects overall dynamics in predator-prey cycles.

The parameterization of juvenile development in the new DEBmodel assuming allocation of a fixed fraction of energy surplus to juvenile growth in the NPM-approach, which is equivalent to assuming $\kappa=(1-M / A) \cdot \lambda_{j u v}+M / A$ for juveniles in a NAM-approach, solves the difficulties arising in NAMs from investment in maturation costs at $\kappa A \leq M$ and $A>M$ without requiring maturity maintenance. The introduction of only one additional parameter i.e. $\lambda_{\text {juv }}$, was sufficient to construct the new DEB-model that has two conceptual advantages compared to a basic NAM: (1) non -starving juveniles only invest into maturation when they can allocate energy to somatic growth and thus can reach maturation, and (2) maturating daphnids of the same size have invested the same amount of energy to reproductive structure; and that better fits the timing of maturation at low food levels than e.g. the NAM of Rinke and Vijverberg (2005).

The improvements by the new energy allocation scheme for juveniles are visible especially at the lowest food levels investigated in the life history experiments on $D$. galeata (Fig. 2) but would not have been detected in the experiments conducted at higher food levels. However, the consequences of the choice of 

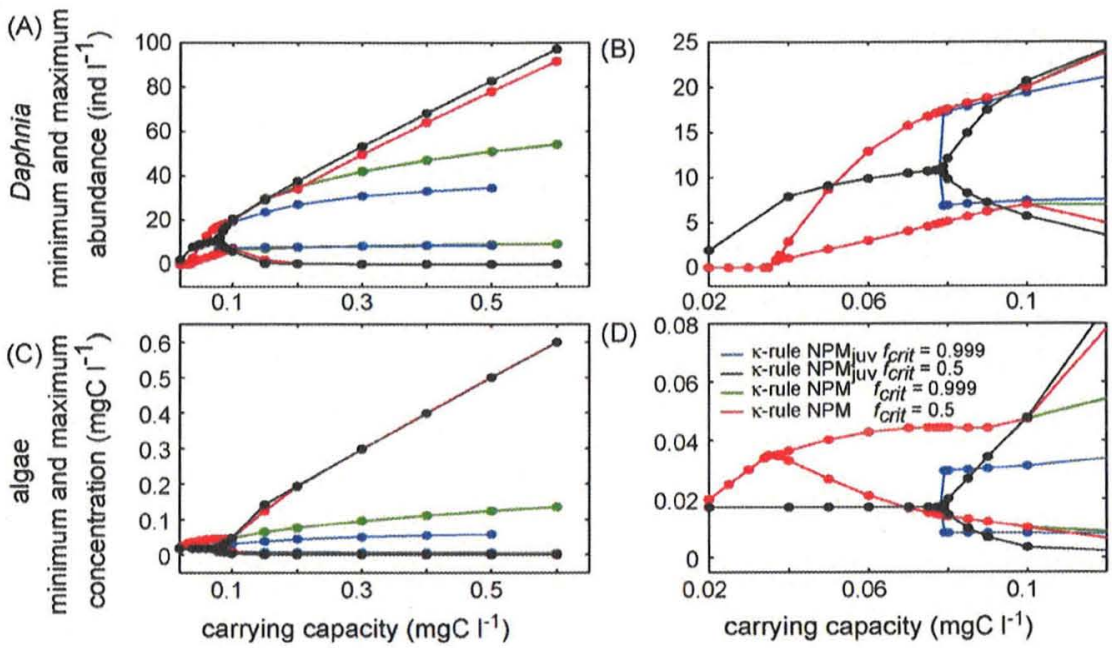

Fig. 10. Bifurcation diagram showing the transition of Daphnia-algae interactions from stable equilibrium to limit cycles with increasing enrichment. The upper panels (A) and B) show minimum and maximum Daphnia abundance during the last 400 days of simulation and (B) gives a detailed view of (A) at low carrying capacities. The lower panels (C) and (D) show maximum and minimum algae concentrations at the same carrying capacities as in (A) and (B), respectively. Simulations with the new DEB-model, i.e. the $\kappa$-rule based NPM with constant $\lambda$ for juveniles ( $\kappa$-rule NPM $\mathrm{N}_{j u v}$, blue and black), are compared to simulations with the $\kappa$-rule based NPM in which the $\kappa$-rule is also applied to juveniles ( $\kappa$-rule NPM, red and green). Two different levels of starving resistance are considered: $f_{\text {crit }}=0.999$ (blue and green) and $f_{\text {crit }}=0.5$ (black and red). Below a carrying capacity of about $0.08 \mathrm{mgCl}^{-1}$ simulations indicated by blue and black are identical and below about $0.2 \mathrm{mgCl}^{-1}$ simulations indicated by red and green are identical. (For interpretation of the references to color in this figure legend, the reader is referred to the web version of the article.)

energy allocation in juveniles become substantial for the dynamics in predator-prey interactions and are particularly important at low food levels. If the $\kappa$-rule energy allocation is applied to juvenile daphnids the range of algae carrying capacities at which daphnids become extinct extends to higher carrying capacities, the stable equilibrium between daphnids and algae occurs for a much narrower range of algal carrying capacities and the Daphnia abundance at stable equilibrium is about $\mathbf{1 0}$ times smaller than in the new DEB-model in which a fixed fraction of net-production is allocated to juvenile growth (Fig. 10B and D). The reason for the substantial differences between the dynamics of the populations with different juvenile energy allocation and the competitive advantage at low food level of the population in which juveniles invest a constant fraction of net-production energy into growth is, that in this allocation scheme juveniles invest more energy into growth at low food levels and therefore reach maturation faster than juveniles following $\kappa$-rule energy allocation.

The physiology under decreasing food levels and especially under starving conditions significantly affects the dynamics of predator-prey interactions (Figs. 7-9). Unfortunately, experimental data on individual physiology under decreasing food levels and starving conditions are very limited making validation of individual models under these conditions impossible. Hence, model predictions on predator-prey dynamics using physiology structured models remain speculative as long as the performance of the individuals during the starving phase in predator-prey cycles are uncertain. Because the population dynamics in predator-prey systems is very sensitive to the physiology under low food and starving conditions the comparison and validation of predator-prey dynamics predicted by physiologically structured population models with observations from laboratory experiments and field data urgently require empirical studies investigating energy allocation under starvation in detail. Without more constraints on the possible physiological strategies under starvation, amplitudes, periods and phases of predator-prey cycles depend on a rather arbitrary choice of e.g. the survivable weight loss parameterized by $f_{\text {crit }}$ that may be adjusted to satisfy observations.

In summary, the new $\kappa$-rule based NPM in which juveniles allocate a fixed fraction of net-production energy to somatic growth $\left(\kappa\right.$-rule $\mathrm{NPM}_{j u v}$ ) improves the modelling of Daphnia life history properties. Simulations based on a structured population model that incorporates the new DEB-model indicate that predator physiology under low food and decreasing food levels has substantial consequences for predator-prey interactions not only affecting the amplitudes and periods of limit cycles but also determining the concentrations of predator and prey at stable equilibrium and the resource range at which stable equilibrium between predator and prey occurs and at which predators become extinct. The results suggests that a physiology based understanding of predator-prey interactions would substantially profit from life history experiments that include the investigation of physiology at low food and under decreasing food levels.

\section{Acknowledgements}

We thank the German Research Foundation (DFG) for financial support of this research ( $\mathrm{Pe} \mathrm{701/2-2} \mathrm{within} \mathrm{the} \mathrm{priority} \mathrm{program}$ 1162 "AQUASHIFT") and Karsten Rinke, and Kristine Schalau for helpful discussions.

\section{Appendix A. Supplementary data}

Supplementary data associated with this article can be found, in the online version, at doi:10.1016/j.ecolmodel.2010.08.006.

\section{References}

Bradley, M.C., Baird, D.J., Calow, P., 1991a. Mechanisms of energy allocation to reproduction in the cladoceran Daphnia magna. Biol. J. Linn. Soc. 44, 325-333.

Bradley, M.C., Perrin, N., Calow, P., 1991b. Energy allocation in the cladoceran Daphnia magna Straus, under starvation and refeeding. Oecologia 86, 414418 .

DeRoos, A.M., Diekmann, O., Metz, J.A.J., 1992. Studying the dynamics of structured population models: a versatile technique and its application to Daphnia. Am. Nat. $139,123-147$

Gliwicz, Z.M., 1990. Food thresholds and body size in cladocerans. Nature 343, $638-640$.

Gliwicz, Z.M., Guisande, C, 1992. Family planning in Daphnia: resistance to starvation in offspring born to mothers grown at different food levels. Oecologia 91, 463-467. 
Gurney, W.S.C, McCauley, E., Nisbet, R.M., Murdoch, W.W., 1990. The physiological ecology of Daphnia: a dynamic model of growth and reproduction. Ecology 71 $716-732$.

Kirk, K.L., 1997. Life-history responses to variable environments: starvation and reproduction in planktonic rotifers. Ecology 78, 434-441.

Kooijman, S.A.L.M., 1986. Population dynamics on basis of energy budgets. In: Metz J.A.J., Diekmann, O. (Eds.), The Dynamics of Physiologically Structured Populations. Springer Verlag, pp. 266-297.

Kooijman, S.A.L.M., 2000. Dynamic Energy and Mass Budgets in Biological Systems. Cambridge University Press.

Kooijman, S.A.L.M., 2001. Quantitative aspects of metabolic organization; a discussion of concepts. Philos. Trans. R. Soc. Lond. Biol. 356, 331-349.

Kooijman, S.A.L.M., 2009. What the egg can tell about its hen: embryonic development on the basis of dynamic energy budgets. I. Math. Biol. 58, 377-394.

Lampert, W., Fleckner, W., Rai, H., Taylor, B.E, 1986. Phytoplankton control by grazing zooplankton: a study on the spring clear-water phase. Limnol. Oceanogr. 31 $478-490$.

Lika, K., Nisbet, R.M., 2000. A dynamic energy budget model based on partitioning of net production. J. Math. Biol. 41, 361 -386.

McCalfey, E., Murdoch, W.M. 1990. Predator-prey dynamics in environment rich and poor in nutrients. Nature 343, 455 457

McCauley, E., Murdoch, W.W., Nisbet, R.M., 1990a. Growth, reproduction, and mortality of Daphnia pulex Leydig: life at low food. Funct. Ecol. 4, 505-514.

McCauley, E., Murdoch, W.W., Nisbet, R.M., Gurney, W.S.C., 1990b. The physiolog ical ecology of Daphnia: development of a model of growth and reproduction. Ecology $71,703-715$

Muller, E.B., Nisbet, R.M., 2000. Survival and production in variable resource environments. Bull. Math. Biol. 62, 1163-1189.

Nisbet, R.M., McCauley, E., Gurney, W.S.C., Murdoch, W.W., Wood, S.N., 2004. Formulating and testing a partially specified dynamic energy budget model. Ecology $85,3132-3139$.

Noonburg, E.G., Nisbet, R.M., McCauley, E., Gurney, W.S.C., Murdoch, W.W., de Roos, A.M., 1998. Experimental testing of dynamic energy budget models. Funct. Ecol $12,211-222$
Plath, K., 1998. Adaptive feeding behavior of Daphnia magna in response to shortterm starvation. Limnol. Oceanogr. 43, 593-599.

Polishchuk, L.V., Vijverberg, J., 2005. Contribution analysis of body mass dynamics in Daphnia. Oecologia 144, 268-277.

Rinke, K., Vijverberg.J., 2005. A model approach to evaluate the effect of temperature and food concentration on individual life-history and population dynamics of Daphnia. Ecol. Model. 186, 326-344.

Robb, G.N. McDonald, R.A., Chamberlain, D.E., Reynolds, S.J., Harrison, T.J.E., Bearhop, S., 2008. Winter feeding of birds increases productivity in the subsequent breed ing season. Biol. Lett. 4, 220-223.

Schalau, K., Rinke, K., Straile, D., Peeters, F., 2008. Temperatur ist the key facto explaining interannual veriability of Daphnia development in spring: a modelling study. Oecologia $157,531-543$.

Tessier, A.J., Henry, L., Goulden, C.E., 1983. Starvation in Daphnia: energy reserves and reproductive allocation. Limnol. Oceanogr. 28, 667-676.

Tessier, A.J., 1986. Comparative population regulation of two planktonic cladocera (Holopedium gibberum and Daphnia catawba). Ecology 67, 285302.

Tyler, N.J.C., Forchhammer, M.C., Oritsland, N.A., 2008. Nonlinear effects of climate and density in the dynamics of a fluctuating population of reindeer. Ecology 89 1675-1686.

Trubetskova, I. Lampert, W, 1995. Egg size and egg mass of Daphnia magna response to food availability. Hydrobiologia 307, 139-145.

Urabe, J., 1988. Effects of food conditions on the net production of Daphnia galeata: separate assessment of growth and reproduction. Bull. Plankton Soc. Jpn. 35 159-174.

van de Wolfshaar, K.E.، de Roos, A.M., Persson, L., 2008. Population feedback after successful invasion leads to ecological suicide in seasonal environments. Ecology $89,259-268$.

Vanoverbeke, J. 2008. Modeling individual and population dynamics in a consumer-resource system: behavior under food limitation and crowding and the effect on population cycling in Daphria. Ecol. Model. 216, 385 401. 